\title{
INVERSION FORMULAS FOR RIEMANN-LIOUVILLE TRANSFORM AND ITS DUAL ASSOCIATED WITH SINGULAR PARTIAL DIFFERENTIAL OPERATORS
}

\author{
C. BACCAR, N. B. HAMADI, AND L. T. RACHDI
}

Received 21 May 2005; Revised 27 September 2005; Accepted 20 October 2005

We define Riemann-Liouville transform $\mathscr{R}_{\alpha}$ and its dual ${ }^{t} \mathscr{R}_{\alpha}$ associated with two singular partial differential operators. We establish some results of harmonic analysis for the Fourier transform connected with $\mathscr{R}_{\alpha}$. Next, we prove inversion formulas for the operators $\mathscr{R}_{\alpha},{ }^{t} \mathscr{R}_{\alpha}$ and a Plancherel theorem for ${ }^{t} \mathscr{R}_{\alpha}$.

Copyright (c) 2006 Hindawi Publishing Corporation. All rights reserved.

\section{Introduction}

The mean operator is defined for a continuous function $f$ on $\mathbb{R}^{2}$, even with respect to the first variable by

$$
\mathfrak{R}_{0}(f)(r, x)=\frac{1}{2 \pi} \int_{0}^{2 \pi} f(r \sin \theta, x+r \cos \theta) d \theta
$$

which means that $\mathfrak{R}_{0}(f)(r, x)$ is the mean value of $f$ on the circle centered at $(0, x)$ and radius $r$. The dual of the mean operator ${ }^{t} \Re_{0}$ is defined by

$$
{ }^{t} \Re_{0}(f)(r, x)=\frac{1}{\pi} \int_{\mathbb{R}} f\left(\sqrt{r^{2}+(x-y)^{2}}, y\right) d y .
$$

The mean operator $\mathfrak{R}_{0}$ and its dual ${ }^{t} \mathfrak{R}_{0}$ play an important role and have many applications, for example, in image processing of the so-called synthetic aperture radar (SAR) data $[11,12]$ or in the linearized inverse scattering problem in acoustics [6].

Our purpose in this work is to define and study integral transforms which generalize the operators $\mathfrak{R}_{0}$ and ${ }^{t} \mathfrak{R}_{0}$. More precisely, we consider the following singular partial differential operators:

$$
\begin{gathered}
\Delta_{1}=\frac{\partial}{\partial x}, \\
\left.\Delta_{2}=\frac{\partial^{2}}{\partial r^{2}}+\frac{2 \alpha+1}{r} \frac{\partial}{\partial r}-\frac{\partial^{2}}{\partial x^{2}}, \quad(r, x) \in\right] 0,+\infty[\times \mathbb{R}, \alpha \geqslant 0 .
\end{gathered}
$$

Hindawi Publishing Corporation

International Journal of Mathematics and Mathematical Sciences

Volume 2006, Article ID 86238, Pages 1-26

DOI 10.1155/IJMMS/2006/86238 
We associate to $\Delta_{1}$ and $\Delta_{2}$ the Riemann-Liouville transform $\Re_{\alpha}$, defined on $\mathscr{C}_{*}\left(\mathbb{R}^{2}\right)$ (the space of continuous functions on $\mathbb{R}^{2}$, even with respect to the first variable) by

$$
\Re_{\alpha}(f)(r, x)=\left\{\begin{array}{rr}
\frac{\alpha}{\pi} \iint_{-1}^{1} f\left(r s \sqrt{1-t^{2}}, x+r t\right) & \\
\times\left(1-t^{2}\right)^{\alpha-1 / 2}\left(1-s^{2}\right)^{\alpha-1} d t d s, & \text { if } \alpha>0, \\
\frac{1}{\pi} \int_{-1}^{1} f\left(r \sqrt{1-t^{2}}, x+r t\right) \frac{d t}{\sqrt{1-t^{2}}}, & \text { if } \alpha=0 .
\end{array}\right.
$$

The dual operator ${ }^{t} \mathfrak{R}_{\alpha}$ is defined on the space $\mathscr{Y}_{*}\left(\mathbb{R}^{2}\right)$ (the space of infinitely differentiable functions on $\mathbb{R}^{2}$, rapidly decreasing together with all their derivatives, even with respect to the first variable) by

$$
{ }^{t} \mathfrak{R}_{\alpha}(f)(r, x)= \begin{cases}\frac{2 \alpha}{\pi} \int_{r}^{+\infty} \int_{-\sqrt{u^{2}-r^{2}}}^{\sqrt{u^{2}-r^{2}}} f(u, x+v)\left(u^{2}-v^{2}-r^{2}\right)^{\alpha-1} u d u d v, & \text { if } \alpha>0, \\ \frac{1}{\pi} \int_{\mathbb{R}} f\left(\sqrt{r^{2}+(x-y)^{2}}, y\right) d y, & \text { if } \alpha=0 .\end{cases}
$$

For more general fractional integrals and fractional differential equations, we can see the works of Debnath [3,4] and Debnath with Bhatta [5].

We establish for the operators $\mathfrak{R}_{\alpha}$ and ${ }^{t} \mathfrak{R}_{\alpha}$ the same results given by Helgason, Ludwig, and Solmon for the classical Radon transform on $\mathbb{R}^{2}[10,14,17]$ and we find the results given in [15] for the spherical mean operator. Especially

(i) we give some harmonic analysis results related to the Fourier transform associated with the Riemann-Liouville transform $\mathfrak{R}_{\alpha}$;

(ii) we define and characterize some spaces of the functions on which $\mathfrak{R}_{\alpha}$ and ${ }^{t} \mathfrak{R}_{\alpha}$ are isomorphisms;

(iii) we give the following inversion formulas for $\mathfrak{R}_{\alpha}$ and ${ }^{t} \mathfrak{R}_{\alpha}$ :

$$
\begin{array}{ll}
f=\mathfrak{R}_{\alpha} K_{\alpha}^{1 t} \mathfrak{R}_{\alpha}(f), & f=K_{\alpha}^{1 t} \mathfrak{R}_{\alpha} \mathfrak{R}_{\alpha}(f), \\
f={ }^{t} \mathfrak{R}_{\alpha} K_{\alpha}^{2} \mathfrak{R}_{\alpha}(f), & f=K_{\alpha}^{2} \mathfrak{R}_{\alpha}{ }^{t} \mathfrak{R}_{\alpha}(f),
\end{array}
$$

where $K_{\alpha}^{1}$ and $K_{\alpha}^{2}$ are integro-differential operators;

(iv) we establish a Plancherel theorem for ${ }^{t} \mathfrak{R}_{\alpha}$;

(v) we show that $\mathfrak{R}_{\alpha}$ and ${ }^{t} \mathfrak{R}_{\alpha}$ are transmutation operators.

This paper is organized as follows. In Section 2, we show that for $(\mu, \lambda) \in \mathbb{C}^{2}$, the differential system

$$
\begin{gathered}
\Delta_{1} u(r, x)=-i \lambda u(r, x), \\
\Delta_{2} u(r, x)=-\mu^{2} u(r, x), \\
u(0,0)=1, \quad \frac{\partial u}{\partial r}(0, x)=0, \quad \forall x \in \mathbb{R},
\end{gathered}
$$


admits a unique solution $\varphi_{\mu, \lambda}$ given by

$$
\varphi_{\mu, \lambda}(r, x)=j_{\alpha}\left(r \sqrt{\mu^{2}+\lambda^{2}}\right) \exp (-i \lambda x),
$$

where $j_{\alpha}$ is the modified Bessel function defined by

$$
j_{\alpha}(s)=2^{\alpha} \Gamma(\alpha+1) \frac{J_{\alpha}(s)}{s^{\alpha}},
$$

and $J_{\alpha}$ is the Bessel function of first kind and index $\alpha$. Next, we prove a Mehler integral representation of $\varphi_{\mu, \lambda}$ and give some properties of $\Re_{\alpha}$.

In Section 3, we define the Fourier transform $\mathfrak{F}_{\alpha}$ connected with $\mathfrak{R}_{\alpha}$, and we establish some harmonic analysis results (inversion formula, Plancherel theorem, Paley-Wiener theorem) which lead to new properties of the operator $\mathfrak{R}_{\alpha}$ and its dual ${ }^{t} \mathfrak{R}_{\alpha}$.

In Section 4 , we characterize some subspaces of $\mathscr{S}_{*}\left(\mathbb{R}^{2}\right)$ on which $\mathfrak{R}_{\alpha}$ and ${ }^{t} \Re_{\alpha}$ are isomorphisms, and we prove the inversion formulas cited below where the operators $K_{\alpha}^{1}$ and $K_{\alpha}^{2}$ are given in terms of Fourier transforms. Next, we introduce fractional powers of the Bessel operator,

$$
\ell_{\alpha}=\frac{\partial^{2}}{\partial r^{2}}+\frac{2 \alpha+1}{r} \frac{\partial}{\partial r}
$$

and the Laplacian operator,

$$
\Delta=\frac{\partial^{2}}{\partial r^{2}}+\frac{\partial^{2}}{\partial x^{2}}
$$

that we use to simplify $K_{\alpha}^{1}$ and $K_{\alpha}^{2}$.

Finally, we prove the following Plancherel theorem for ${ }^{t} \mathfrak{R}_{\alpha}$ :

$$
\int_{\mathbb{R}} \int_{0}^{+\infty}|f(r, x)|^{2} r^{2 \alpha+1} d r d x=\int_{\mathbb{R}} \int_{0}^{+\infty}\left|K_{\alpha}^{3}\left({ }^{t} \Re_{\alpha}(f)\right)(r, x)\right|^{2} d r d x
$$

where $K_{\alpha}^{3}$ is an integro-differential operator.

In Section 5, we show that $\mathfrak{R}_{\alpha}$ and ${ }^{t} \Re_{\alpha}$ satisfy the following relations of permutation:

$$
\begin{aligned}
& { }^{t} \mathfrak{R}_{\alpha}\left(\Delta_{2} f\right)=\frac{\partial^{2}}{\partial r^{2}}{ }^{t} \mathfrak{R}_{\alpha}(f), \quad{ }^{t} \mathfrak{R}_{\alpha}\left(\Delta_{1} f\right)=\Delta_{1}{ }^{t} \mathfrak{R}_{\alpha}(f), \\
& \Delta_{2} \mathfrak{R}_{\alpha}(f)=\mathfrak{R}_{\alpha}\left(\frac{\partial^{2} f}{\partial r^{2}}\right), \quad \Delta_{1} \mathfrak{R}_{\alpha}(f)=\mathfrak{R}_{\alpha}\left(\Delta_{1} f\right) .
\end{aligned}
$$

\section{Riemann-Liouville transform and its dual associated with the operators $\Delta_{1}$ and $\Delta_{2}$}

In this section, we define the Riemann-Liouville transform $\mathfrak{R}_{\alpha}$ and its dual ${ }^{t} \mathfrak{R}_{\alpha}$, and we give some properties of these operators. It is well known [21] that for every $\lambda \in \mathbb{C}$, the system

$$
\begin{gathered}
\ell_{\alpha} v(r)=-\lambda^{2} v(r) ; \\
v(0)=1 ; \quad v^{\prime}(0)=0,
\end{gathered}
$$


4 Inversion formulas for Riemann-Liouville transform

where $\ell_{\alpha}$ is the Bessel operator, admits a unique solution, that is, the modified Bessel function $r \mapsto j_{\alpha}(r \lambda)$. Thus, for all $(\mu, \lambda) \in \mathbb{C} \times \mathbb{C}$, the system

$$
\begin{gathered}
\Delta_{1} u(r, x)=-i \lambda u(r, x), \\
\Delta_{2} u(r, x)=-\mu^{2} u(r, x), \\
u(0,0)=1, \quad \frac{\partial u}{\partial r}(0, x)=0, \quad \forall x \in \mathbb{R},
\end{gathered}
$$

admits the unique solution given by

$$
\varphi_{\mu, \lambda}(r, x)=j_{\alpha}\left(r \sqrt{\mu^{2}+\lambda^{2}}\right) \exp (-i \lambda x)
$$

The modified Bessel function $j_{\alpha}$ has the Mehler integral representation, (we refer to $[13,21])$

$$
j_{\alpha}(s)=\frac{\Gamma(\alpha+1)}{\sqrt{\pi} \Gamma(\alpha+1 / 2)} \int_{-1}^{1}\left(1-t^{2}\right)^{\alpha-1 / 2} \exp (-i s t) d t .
$$

In particular,

$$
\forall k \in \mathbb{N}, \forall s \in \mathbb{R}, \quad\left|j_{\alpha}^{(k)}(s)\right| \leqslant 1
$$

On the other hand,

$$
\sup _{r \in \mathbb{R}}\left|j_{\alpha}(r \lambda)\right|=1 \quad \text { iff } \lambda \in \mathbb{R} \text {. }
$$

This involves that

$$
\sup _{(r, x) \in \mathbb{R}^{2}}\left|\varphi_{\mu, \lambda}(r, x)\right|=1 \quad \text { iff }(\mu, \lambda) \in \Gamma,
$$

where $\Gamma$ is the set defined by

$$
\Gamma=\mathbb{R}^{2} \cup\left\{(i \mu, \lambda) ;(\mu, \lambda) \in \mathbb{R}^{2},|\mu| \leqslant|\lambda|\right\} .
$$

Proposition 2.1. The eigenfunction $\varphi_{\mu, \lambda}$ given by (2.3) has the following Mehler integral representation:

$$
\varphi_{\mu, \lambda}(r, x)= \begin{cases}\frac{\alpha}{\pi} \iint_{-1}^{1} \cos \left(\mu r s \sqrt{1-t^{2}}\right) \exp (-i \lambda(x+r t))\left(1-t^{2}\right)^{\alpha-1 / 2}\left(1-s^{2}\right)^{\alpha-1} d t d s, \\ \frac{1}{\pi} \int_{-1}^{1} \cos \left(r \mu \sqrt{1-t^{2}}\right) \exp (-i \lambda(x+r t)) \frac{d t}{\sqrt{1-t^{2}}}, & \text { if } \alpha=0 .\end{cases}
$$


Proof. From the following expansion of the function $j_{\alpha}$ :

$$
j_{\alpha}(s)=2^{\alpha} \Gamma(\alpha+1) \frac{J_{\alpha}(s)}{s^{\alpha}}=\Gamma(\alpha+1) \sum_{k=0}^{+\infty} \frac{(-1)^{k}}{k ! \Gamma(\alpha+k+1)}\left(\frac{s}{2}\right)^{2 k}
$$

we deduce that

$$
j_{\alpha}\left(r \sqrt{\mu^{2}+\lambda^{2}}\right)=\Gamma(\alpha+1) \sum_{k=0}^{+\infty} \frac{(-1)^{k}}{k ! \Gamma(k+\alpha+1)}\left(\frac{r \mu}{2}\right)^{2 k} j_{\alpha+k}(r \lambda),
$$

and from the equality (2.4), we obtain

$$
j_{\alpha}\left(r \sqrt{\mu^{2}+\lambda^{2}}\right)=\frac{\Gamma(\alpha+1)}{\sqrt{\pi} \Gamma(\alpha+1 / 2)} \int_{-1}^{1} j_{\alpha-1 / 2}\left(r \mu \sqrt{1-t^{2}}\right) \exp (-i r \lambda t)\left(1-t^{2}\right)^{\alpha-1 / 2} d t .
$$

Then, the results follow by using again the relation (2.4) for $\alpha>0$, and from the fact that

$$
j_{-1 / 2}(s)=\cos s, \quad \text { for } \alpha=0 \text {. }
$$

Definition 2.2. The Riemann-Liouville transform $\mathfrak{R}_{\alpha}$ associated with the operators $\Delta_{1}$ and $\Delta_{2}$ is the mapping defined on $\mathscr{C}_{*}\left(\mathbb{R}^{2}\right)$ by the following. For all $(r, x) \in \mathbb{R}^{2}$,

$$
\Re_{\alpha}(f)(r, x)=\left\{\begin{array}{rr}
\frac{\alpha}{\pi} \iint_{-1}^{1} f\left(r s \sqrt{1-t^{2}}, x+r t\right) & \\
\times\left(1-t^{2}\right)^{\alpha-1 / 2}\left(1-s^{2}\right)^{\alpha-1} d t d s, & \text { if } \alpha>0, \\
\frac{1}{\pi} \int_{-1}^{1} f\left(r \sqrt{1-t^{2}}, x+r t\right) \frac{d t}{\sqrt{1-t^{2}}}, & \text { if } \alpha=0 .
\end{array}\right.
$$

Remark 2.3. (i) From Proposition 2.1 and Definition 2.2, we have

$$
\varphi_{\mu, \lambda}(r, x)=\Re_{\alpha}(\cos (\mu .) \exp (-i \lambda .))(r, x)
$$

(ii) We can easily see, as in [2], that the transform $\Re_{\alpha}$ is continuous and injective from $\mathscr{E}_{*}\left(\mathbb{R}^{2}\right)$ (the space of infinitely differentiable functions on $\mathbb{R}^{2}$, even with respect to the first variable) into itself. 
6 Inversion formulas for Riemann-Liouville transform

Lemma 2.4. For $f \in \mathscr{C}_{*}\left(\mathbb{R}^{2}\right), f$ bounded, and $g \in \mathscr{Y}_{*}\left(\mathbb{R}^{2}\right)$,

$$
\int_{\mathbb{R}} \int_{0}^{+\infty} \mathfrak{R}_{\alpha}(f)(r, x) g(r, x) r^{2 \alpha+1} d r d x=\int_{\mathbb{R}} \int_{0}^{+\infty} f(r, x){ }^{t} \mathfrak{R}_{\alpha}(g)(r, x) d r d x,
$$

where ${ }^{t} \mathfrak{R}_{\alpha}$ is the dual transform defined by

$$
{ }^{t} \mathfrak{R}_{\alpha}(g)(r, x)= \begin{cases}\frac{2 \alpha}{\pi} \int_{r}^{+\infty} \int_{-\sqrt{u^{2}-r^{2}}}^{\sqrt{u^{2}-r^{2}}} g(u, x+v)\left(u^{2}-v^{2}-r^{2}\right)^{\alpha-1} u d u d v, & \text { if } \alpha>0, \\ \frac{1}{\pi} \int_{\mathbb{R}} g\left(\sqrt{r^{2}+(x-y)^{2}}, y\right) d y, & \text { if } \alpha=0 .\end{cases}
$$

To obtain this lemma, we use Fubini's theorem and an adequate change of variables. Remark 2.5. By a simple change of variables, we have

$$
\Re_{0}(f)(r, x)=\frac{1}{2 \pi} \int_{0}^{2 \pi} f(r \sin \theta, x+r \cos \theta) d \theta .
$$

\section{Fourier transform associated with Riemann-Liouville operator}

In this section, we define the Fourier transform associated with the operator $\mathfrak{R}_{\alpha}$, and we give some results of harmonic analysis that we use in the next sections.

We denote by

(i) $d v(r, x)$ the measure defined on $[0,+\infty[\times \mathbb{R}$ by

$$
d \nu(r, x)=\frac{1}{\sqrt{2 \pi} 2^{\alpha} \Gamma(\alpha+1)} r^{2 \alpha+1} d r \otimes d x,
$$

(ii) $L^{1}(d \nu)$ the space of measurable functions $f$ on $[0,+\infty[\times \mathbb{R}$ satisfying

$$
\|f\|_{1, \nu}=\int_{\mathbb{R}} \int_{0}^{+\infty}|f(r, x)| d \nu(r, x)<+\infty .
$$

Definition 3.1. (i) The translation operator associated with Riemann-Liouville transform is defined on $L^{1}(d v)$ by the following. For all $(r, x),(s, y) \in[0,+\infty[\times \mathbb{R}$,

$$
\mathscr{T}_{(r, x)} f(s, y)=\frac{\Gamma(\alpha+1)}{\sqrt{\pi} \Gamma(\alpha+1 / 2)} \int_{0}^{\pi} f\left(\sqrt{r^{2}+s^{2}+2 r s \cos \theta}, x+y\right) \sin ^{2 \alpha} \theta d \theta .
$$

(ii) The convolution product associated with the Riemann-Liouville transform of $f$, $g \in L^{1}(d \nu)$ is defined by the following. For all $(r, x) \in[0,+\infty[\times \mathbb{R}$,

$$
f \# g(r, x)=\int_{\mathbb{R}} \int_{0}^{+\infty} \mathcal{T}_{(r,-x)} \check{f}(s, y) g(s, y) d \nu(s, y),
$$

where $\check{f}(s, y)=f(s,-y)$. 
We have the following properties.

(i) Since

$$
\forall r, s \geqslant 0, \quad j_{\alpha}(r \lambda) j_{\alpha}(s \lambda)=\frac{\Gamma(\alpha+1)}{\sqrt{\pi} \Gamma(\alpha+1 / 2)} \int_{0}^{\pi} j_{\alpha}\left(\lambda \sqrt{r^{2}+s^{2}+2 r s \cos \theta}\right) \sin ^{2 \alpha} \theta d \theta,
$$

(we refer to [21]) we deduce that the eigenfunction $\varphi_{\mu, \lambda}$ defined by the relation (2.3) satisfies the product formula

$$
\mathscr{T}_{(r, x)} \varphi_{\mu, \lambda}(s, y)=\varphi_{\mu, \lambda}(r, x) \varphi_{\mu, \lambda}(s, y) .
$$

(ii) If $f \in L^{1}(d v)$, then for all $(r, x) \in\left[0,+\infty\left[\times \mathbb{R}, \mathscr{T}_{(r, x)} f\right.\right.$ belongs to $L^{1}(d v)$, and we have

$$
\left\|\mathscr{T}_{(r, x)} f\right\|_{1, \nu} \leqslant\|f\|_{1, \nu}
$$

(iii) For $f, g \in L^{1}(d v), f \# g$ belongs to $L^{1}(d v)$, and the convolution product is commutative and associative.

(iv) For $f, g \in L^{1}(d v)$,

$$
\|f \# g\|_{1, \nu} \leqslant\|f\|_{1, \nu}\|g\|_{1, \nu}
$$

Definition 3.2. The Fourier transform associated with the Riemann-Liouville operator is defined by

$$
\forall(\mu, \lambda) \in \Gamma, \quad \mathfrak{F}_{\alpha}(f)(\mu, \lambda)=\int_{\mathbb{R}} \int_{0}^{+\infty} f(r, x) \varphi_{\mu, \lambda}(r, x) d \nu(r, x),
$$

where $\Gamma$ is the set defined by the relation (2.8).

We have the following properties.

(i) Let $f$ be in $L^{1}(d \nu)$. For all $(r, x) \in[0,+\infty[\times \mathbb{R}$, we have

$$
\forall(\mu, \lambda) \in \Gamma, \quad \mathfrak{F}_{\alpha}\left(\mathscr{T}_{(r,-x)} f\right)(\mu, \lambda)=\varphi_{\mu, \lambda}(r, x) \mathfrak{F}_{\alpha}(f)(\mu, \lambda)
$$

(ii) For $f, g \in L^{1}(d \nu)$, we have

$$
\forall(\mu, \lambda) \in \Gamma, \quad \mathfrak{F}_{\alpha}(f \# g)(\mu, \lambda)=\mathfrak{F}_{\alpha}(f)(\mu, \lambda) \mathfrak{F}_{\alpha}(g)(\mu, \lambda)
$$

(iii) For $f \in L^{1}(d \nu)$, we have

$$
\forall(\mu, \lambda) \in \Gamma, \quad \mathfrak{F}_{\alpha}(f)(\mu, \lambda)=B \circ \widetilde{\mathfrak{F}}_{\alpha}(f)(\mu, \lambda),
$$

where

$$
\begin{gathered}
\forall(\mu, \lambda) \in \mathbb{R}^{2}, \quad \widetilde{\mathfrak{F}}_{\alpha}(f)(\mu, \lambda)=\int_{\mathbb{R}} \int_{0}^{+\infty} f(r, x) j_{\alpha}(r \mu) \exp (-i \lambda x) d \nu(r, x), \\
\forall(\mu, \lambda) \in \Gamma, \quad B f(\mu, \lambda)=f\left(\sqrt{\mu^{2}+\lambda^{2}}, \lambda\right) .
\end{gathered}
$$


8 Inversion formulas for Riemann-Liouville transform

3.1. Inversion formula and Plancherel theorem for $\mathfrak{F}_{\alpha}$. We denote by (see [15])

(i) $\mathscr{S}_{*}\left(\mathbb{R}^{2}\right)$ the space of infinitely differentiable functions on $\mathbb{R}^{2}$ rapidly decreasing together with all their derivatives, even with respect to the first variable;

(ii) $\mathscr{I}_{*}(\Gamma)$ the space of functions $f: \Gamma \rightarrow \mathbb{C}$ infinitely differentiable, even with respect to the first variable and rapidly decreasing together with all their derivatives, that is, for all $k_{1}, k_{2}, k_{3} \in \mathbb{N}$,

$$
\sup _{(\mu, \lambda) \in \Gamma}\left(1+|\mu|^{2}+|\lambda|^{2}\right)^{k_{1}}\left|\left(\frac{\partial}{\partial \mu}\right)^{k_{2}}\left(\frac{\partial}{\partial \lambda}\right)^{k_{3}} f(\mu, \lambda)\right|<+\infty,
$$

where

$$
\frac{\partial f}{\partial \mu}(\mu, \lambda)= \begin{cases}\frac{\partial}{\partial r}(f(r, \lambda)), & \text { if } \mu=r \in \mathbb{R} \\ \frac{1}{i} \frac{\partial}{\partial t}(f(i t, \lambda)), & \text { if } \mu=i t,|t| \leqslant|\lambda|\end{cases}
$$

Each of these spaces is equipped with its usual topology:

(i) $L^{2}(d v)$ the space of measurable functions on $[0,+\infty[\times \mathbb{R}$ such that

$$
\|f\|_{2, \nu}=\left(\int_{\mathbb{R}} \int_{0}^{+\infty}|f(r, x)|^{2} d \nu(r, x)\right)^{1 / 2}<+\infty ;
$$

(ii) $d \gamma(\mu, \lambda)$ the measure defined on $\Gamma$ by

$$
\begin{aligned}
& \iint_{\Gamma} f(\mu, \lambda) d \gamma(\mu, \lambda) \\
& =\frac{1}{\sqrt{2 \pi} 2^{\alpha} \Gamma(\alpha+1)}\left\{\int_{\mathbb{R}} \int_{0}^{+\infty} f(\mu, \lambda)\left(\mu^{2}+\lambda^{2}\right)^{\alpha} \mu d \mu d \lambda+\int_{\mathbb{R}} \int_{0}^{|\lambda|} f(i \mu, \lambda)\left(\lambda^{2}-\mu^{2}\right)^{\alpha} \mu d \mu d \lambda\right\}
\end{aligned}
$$

(iii) $L^{p}(d \gamma), p=1, p=2$, the space of measurable functions on $\Gamma$ satisfying

$$
\|f\|_{p, \gamma}=\left(\iint_{\Gamma}|f(\mu, \lambda)|^{p} d \gamma(\mu, \lambda)\right)^{1 / p}<+\infty .
$$

Remark 3.3. It is clear that a function $f$ belongs to $L^{1}(d \nu)$ if, and only if, the function $B f$ belongs to $L^{1}(d \gamma)$, and we have

$$
\iint_{\Gamma} B f(\mu, \lambda) d \gamma(\mu, \lambda)=\int_{\mathbb{R}} \int_{0}^{+\infty} f(r, x) d \nu(r, x) .
$$

Proposition 3.4 (inversion formula for $\mathfrak{F}_{\alpha}$ ). Let $f \in L^{1}(d \nu)$ such that $\mathfrak{F}_{\alpha}(f)$ belongs to $L^{1}(d \gamma)$, then for almost every $(r, x) \in[0,+\infty[\times \mathbb{R}$,

$$
f(r, x)=\iint_{\Gamma} \mathfrak{F}_{\alpha}(f)(\mu, \lambda) \bar{\varphi}_{\mu, \lambda}(r, x) d \gamma(\mu, \lambda) .
$$


Proof. From $[9,19]$, one can see that if $f \in L^{1}(d \nu)$ is such that $\widetilde{\mathfrak{F}}_{\alpha}(f) \in L^{1}(d \nu)$, then for almost every $(r, x) \in[0,+\infty[\times \mathbb{R}$,

$$
f(r, x)=\int_{\mathbb{R}} \int_{0}^{+\infty} \widetilde{\mathfrak{F}}_{\alpha}(f)(\mu, \lambda) j_{\alpha}(r \mu) \exp (i \lambda x) d \nu(\mu, \lambda)
$$

Then, the result follows from the relation (3.12) and Remark 3.3.

Theorem 3.5. (i) The Fourier transform $\mathfrak{F}_{\alpha}$ is an isomorphism from $\mathscr{S}_{*}\left(\mathbb{R}^{2}\right)$ onto $\mathscr{Y}_{*}(\Gamma)$.

(ii) (Plancherel formula) for $f \in \mathscr{S}_{*}\left(\mathbb{R}^{2}\right)$,

$$
\left\|\mathfrak{F}_{\alpha}(f)\right\|_{2, \gamma}=\|f\|_{2, \nu}
$$

(iii) (Plancherel theorem) the transform $\mathfrak{F}_{\alpha}$ can be extended to an isometric isomorphism from $L^{2}(d \nu)$ onto $L^{2}(d \gamma)$.

Proof. This theorem follows from the relation (3.12), Remark 3.3, and the fact that $\widetilde{\mathfrak{F}}_{\alpha}$ is an isomorphism from $\mathscr{S}_{*}\left(\mathbb{R}^{2}\right)$ onto itself, satisfying that for all $f \in \mathscr{S}_{*}\left(\mathbb{R}^{2}\right)$,

$$
\left\|\widetilde{\mathfrak{F}}_{\alpha}(f)\right\|_{2, \nu}=\|f\|_{2, \nu}
$$

Lemma 3.6. For $f \in \mathscr{Y}_{*}\left(\mathbb{R}^{2}\right)$,

$$
\forall(\mu, \lambda) \in \mathbb{R}^{2}, \quad \mathfrak{F}_{\alpha}(f)(\mu, \lambda)=\Lambda_{\alpha} \circ{ }^{t} \mathfrak{R}_{\alpha}(f)(\mu, \lambda),
$$

where ${ }^{t} \Re_{\alpha}$ is the dual transform of the Riemann-Liouville operator, and $\Lambda_{\alpha}$ is a constant multiple of the classical Fourier transform on $\mathbb{R}^{2}$ defined by

$$
\Lambda_{\alpha}(f)(\mu, \lambda)=\int_{\mathbb{R}} \int_{0}^{+\infty} f(r, x) \cos (r \mu) \exp (-i \lambda x) d m(r, x),
$$

where $d m(r, x)$ is the measure defined on $[0,+\infty[\times \mathbb{R}$ by

$$
d m(r, x)=\frac{1}{\sqrt{2 \pi} 2^{\alpha} \Gamma(\alpha+1)} d r \otimes d x
$$

This lemma follows from the relation (2.15) and Lemma 2.4.

Using the relation (3.12) and the fact that the mapping $B$ is continuous from $\mathscr{Y}_{*}\left(\mathbb{R}^{2}\right)$ into itself, we deduce that the Fourier transform $\mathfrak{F}_{\alpha}$ is continuous from $\mathscr{Y}_{*}\left(\mathbb{R}^{2}\right)$ into itself. On the other hand, $\Lambda_{\alpha}$ is an isomorphism from $\mathscr{S}_{*}\left(\mathbb{R}^{2}\right)$ onto itself. Then, Lemma 3.6 implies that the dual transform ${ }^{t} \Re_{\alpha}$ maps continuously $\mathscr{I}_{*}\left(\mathbb{R}^{2}\right)$ into itself.

Proposition 3.7. (i) ${ }^{t} \mathfrak{R}_{\alpha}$ is not injective when applied to $\mathscr{S}_{*}\left(\mathbb{R}^{2}\right)$.

(ii) ${ }^{t} \mathfrak{R}_{\alpha}\left(\mathscr{S}_{*}\left(\mathbb{R}^{2}\right)\right)=\mathscr{S}_{*}\left(\mathbb{R}^{2}\right)$. 
Proof. (i) Let $g \in \mathscr{I}_{*}\left(\mathbb{R}^{2}\right)$ such that supp $g \subset\left\{(r, x) \in \mathbb{R}^{2},|r| \leqslant|x|\right\}, g \neq 0$.

Since $\widetilde{\mathfrak{F}}_{\alpha}$ is an isomorphism from $\mathscr{S}_{*}\left(\mathbb{R}^{2}\right)$ onto itself, there exists $f \in \mathscr{Y}_{*}\left(\mathbb{R}^{2}\right)$ such that $\widetilde{\mathfrak{F}}_{\alpha}(f)=g$. From the relation (3.12) and Lemma 3.6, we deduce that ${ }^{t} \mathfrak{R}_{\alpha}(f)=0$.

(ii) We obtain the result by the same way as in [1].

\subsection{Paley-Wiener theorem. We denote by}

(i) $\mathscr{D}_{*}\left(\mathbb{R}^{2}\right)$ the space of infinitely differentiable functions on $\mathbb{R}^{2}$, even with respect to the first variable, and with compact support;

(ii) $\mathbb{U}_{*}\left(\mathbb{C}^{2}\right)$ the space of entire functions $f: \mathbb{C}^{2} \rightarrow \mathbb{C}$, even with respect to the first variable rapidly decreasing of exponential type, that is, there exists a positive constant $M$, such that for all $k \in \mathbb{N}$,

$$
\sup _{(\mu, \lambda) \in \mathbb{C}^{2}}\left(1+|\mu|^{2}+|\lambda|^{2}\right)^{k}|f(\mu, \lambda)| \exp (-M(|\operatorname{Im} \mu|+|\operatorname{Im} \lambda|))<+\infty
$$

(iii) $\mathbb{H}_{*, 0}\left(\mathbb{C}^{2}\right)$ the subspace of $\mathbb{H}_{*}\left(\mathbb{C}^{2}\right)$, consisting of functions $f: \mathbb{C}^{2} \rightarrow \mathbb{C}$, such that for all $k \in \mathbb{N}$,

$$
\sup _{\substack{(\mu, \lambda) \in \mathbb{R}^{2} \\|\mu| \leqslant|\lambda|}}\left(1-\mu^{2}+2 \lambda^{2}\right)^{k}|f(i \mu, \lambda)|<+\infty
$$

(iv) $\mathscr{E}_{*}^{\prime}\left(\mathbb{R}^{2}\right)$ the space of distributions on $\mathbb{R}^{2}$, even with respect to the first variable, and with compact support;

(v) $\mathscr{H}_{*}\left(\mathbb{C}^{2}\right)$ the space of entire functions $f: \mathbb{C}^{2} \rightarrow \mathbb{C}$, even with respect to the first variable, slowly increasing of exponential type, that is, there exist a positive constant $M$ and an integer $k$, such that

$$
\sup _{(\mu, \lambda) \in \mathbb{C}^{2}}\left(1+|\mu|^{2}+|\lambda|^{2}\right)^{-k}|f(\mu, \lambda)| \exp (-M(|\operatorname{Im} \mu|+|\operatorname{Im} \lambda|))<+\infty
$$

(vi) $\mathscr{H}_{*, 0}\left(\mathbb{C}^{2}\right)$ the subspace of $\mathscr{H}_{*}\left(\mathbb{C}^{2}\right)$, consisting of functions $f: \mathbb{C}^{2} \rightarrow \mathbb{C}$, such that there exists an integer $k$, satisfying

$$
\sup _{\substack{(\mu, \lambda) \in \mathbb{R}^{2} \\|\mu| \leqslant|\lambda|}}\left(1-\mu^{2}+2 \lambda^{2}\right)^{-k}|f(i \mu, \lambda)|<+\infty .
$$

Each of these spaces is equipped with its usual topology.

Definition 3.8. The Fourier transform associated with the Riemann-Liouville operator is defined on $\mathscr{E}_{*}^{\prime}\left(\mathbb{R}^{2}\right)$ by

$$
\forall(\mu, \lambda) \in \mathbb{C}^{2}, \quad \mathfrak{F}_{\alpha}(T)(\mu, \lambda)=\left\langle T, \varphi_{\mu, \lambda}\right\rangle
$$


Proposition 3.9. For every $T \in \mathscr{E}_{*}^{\prime}\left(\mathbb{R}^{2}\right)$,

$$
\forall(\mu, \lambda) \in \mathbb{C}^{2}, \quad \mathfrak{F}_{\alpha}(T)(\mu, \lambda)=B \circ \widetilde{\mathfrak{F}}_{\alpha}(T)(\mu, \lambda)
$$

where

$$
\forall(\mu, \lambda) \in \mathbb{C}^{2}, \quad \widetilde{\mathfrak{F}}_{\alpha}(T)(\mu, \lambda)=\left\langle T, j_{\alpha}(\mu .) \exp (-i \lambda .)\right\rangle
$$

and $B$ is the transform defined by the relation (3.12).

Using [7, Lemma 2] (see also [15]) and the fact that $\widetilde{\mathfrak{F}}_{\alpha}$ is an isomorphism from $\mathscr{D}_{*}\left(\mathbb{R}^{2}\right)\left(\right.$ resp., $\left.\mathscr{E}_{*}^{\prime}\left(\mathbb{R}^{2}\right)\right)$ onto $\mathbb{U} *\left(\mathbb{C}^{2}\right)$ (resp., $\mathscr{H}_{*}\left(\mathbb{C}^{2}\right)$ ), we deduce the following theorem.

Theorem 3.10 (of Paley-Wiener). The Fourier transform $\mathfrak{F}_{\alpha}$ is an isomorphism

(i) from $\mathscr{D}_{*}\left(\mathbb{R}^{2}\right)$ onto $\mathbb{U}_{*, 0}\left(\mathbb{C}^{2}\right)$;

(ii) from $\mathscr{E}_{*}^{\prime}\left(\mathbb{R}^{2}\right)$ onto $\mathscr{H}_{*, 0}\left(\mathbb{C}^{2}\right)$.

From Lemma 3.6, Theorem 3.10, and the fact that $\Lambda_{\alpha}$ is an isomorphism from $\mathscr{D}_{*}\left(\mathbb{R}^{2}\right)$ onto $\mathbb{H}_{*}\left(\mathbb{C}^{2}\right)$, we have the following corollary.

Corollary 3.11. (i) ${ }^{t} \mathfrak{R}_{\alpha}$ maps injectively $\mathscr{D}_{*}\left(\mathbb{R}^{2}\right)$ into itself.

(ii) ${ }^{t} \mathfrak{R}_{\alpha}\left(\mathscr{D}_{*}\left(\mathbb{R}^{2}\right)\right) \neq \mathscr{D}_{*}\left(\mathbb{R}^{2}\right)$.

\section{Inversion formulas for $\mathfrak{R}_{\alpha}$ and ${ }^{t} \mathfrak{R}_{\alpha}$ and Plancherel theorem for ${ }^{t} \mathfrak{R}_{\alpha}$}

In this section, we will define some subspaces of $\mathscr{Y}_{*}\left(\mathbb{R}^{2}\right)$ on which $\mathfrak{R}_{\alpha}$ and ${ }^{t} \mathfrak{R}_{\alpha}$ are isomorphisms, and we give their inverse transforms in terms of integro-differential operators. Next, we establish Plancherel theorem for ${ }^{t} \Re_{\alpha}$.

We denote by

(i) $\mathcal{N}$ the subspace of $\mathscr{Y}_{*}\left(\mathbb{R}^{2}\right)$, consisting of functions $f$ satisfying

$$
\forall k \in \mathbb{N}, \forall x \in \mathbb{R}, \quad\left(\frac{\partial}{\partial r^{2}}\right)^{k} f(0, x)=0,
$$

where

$$
\frac{\partial}{\partial r^{2}}=\frac{1}{r} \frac{\partial}{\partial r}
$$

(ii) $\mathscr{Y}_{*, 0}\left(\mathbb{R}^{2}\right)$ the subspace of $\mathscr{Y}_{*}\left(\mathbb{R}^{2}\right)$, consisting of functions $f$, such that

$$
\forall k \in \mathbb{N}, \forall x \in \mathbb{R}, \quad \int_{0}^{+\infty} f(r, x) r^{2 k} d r=0 ;
$$

(iii) $\mathscr{S}_{*}^{0}\left(\mathbb{R}^{2}\right)$ the subspace of $\mathscr{Y}_{*}\left(\mathbb{R}^{2}\right)$, consisting of functions $f$, such that

$$
\operatorname{supp} \widetilde{\mathfrak{F}}_{\alpha}(f) \subset\left\{(\mu, \lambda) \in \mathbb{R}^{2} ;|\mu| \geqslant|\lambda|\right\} .
$$


12 Inversion formulas for Riemann-Liouville transform

Lemma 4.1. (i) The mapping $\Lambda_{\alpha}$ is an isomorphism from $\mathscr{Y}_{*, 0}\left(\mathbb{R}^{2}\right)$ onto $\mathcal{N}$.

(ii) The subspace $\mathcal{N}$ can be written as

$$
\mathcal{N}=\left\{f \in \mathscr{Y}_{*}\left(\mathbb{R}^{2}\right) ; \forall k \in \mathbb{N}, \forall x \in \mathbb{R} ;\left(\frac{\partial}{\partial r}\right)^{2 k} f(0, x)=0\right\}
$$

Proof. Let $f \in \mathscr{Y}_{*, 0}\left(\mathbb{R}^{2}\right)$.

(i) For $v>-1$, we have

$$
\left(\frac{\partial}{\partial \mu^{2}}\right)^{k}\left(j_{\nu}(r \mu)\right)=\frac{\Gamma(\nu+1)}{2^{k} \Gamma(\nu+k+1)}\left(-r^{2}\right)^{k} j_{\nu+k}(r \mu),
$$

thus, from the expression of $\Lambda_{\alpha}$, given in Lemma 3.6, and the fact that $j_{-1 / 2}(s)=\cos s$, we obtain

$$
\left(\frac{\partial}{\partial \mu^{2}}\right)^{k}\left(\Lambda_{\alpha}(f)\right)(0, \lambda)=\frac{\sqrt{\pi}}{2^{k} \Gamma(k+1 / 2)}(-1)^{k} \int_{\mathbb{R}} \int_{0}^{+\infty} f(r, x) r^{2 k} \exp (-i \lambda x) d m(r, x),
$$

which gives the result.

(ii) The proof of (ii) is immediate.

Theorem 4.2. (i) For all real numbers $\gamma$, the mappings

(i) $f \mapsto\left(r^{2}+x^{2}\right)^{\gamma} f$

(ii) $f \mapsto|r|^{\gamma} f$

are isomorphisms from $\mathcal{N}$ onto itself.

(ii) For $f \in \mathcal{N}$, the function $g$ defined by

$$
g(r, x)= \begin{cases}f\left(\sqrt{r^{2}-x^{2}}, x\right) & \text { if }|r| \geqslant|x| \\ 0 & \text { otherwise }\end{cases}
$$

belongs to $\mathscr{S}_{*}\left(\mathbb{R}^{2}\right)$.

Proof. (i) Let $f \in \mathcal{N}$, by Leibnitz formula, we have

$$
\begin{aligned}
\left(\frac{\partial}{\partial r}\right)^{k_{1}} & \left(\frac{\partial}{\partial x}\right)^{k_{2}}\left[\left(r^{2}+x^{2}\right)^{\gamma} f\right](r, x) \\
& =\sum_{j=0}^{k_{1}} \sum_{i=0}^{k_{2}} C_{k_{1}}^{j} C_{k_{2}}^{i} P_{j}(r) P_{i}(x)\left(r^{2}+x^{2}\right)^{\gamma-i-j} \frac{\partial^{k_{1}+k_{2}-i-j}}{\partial r^{k_{1}-j} \partial x^{k_{2}-i}} f(r, x)
\end{aligned}
$$

where $P_{i}$ and $P_{j}$ are real polynomials. 
Let $n \in \mathbb{N}$ such that $\gamma-k_{1}-k_{2}+n>0$. By Taylor formula and the fact that $f \in \mathcal{N}$, we have

$$
\begin{aligned}
\left(\frac{\partial}{\partial r}\right)^{k_{1}-j}(f)(r, x) & =\frac{r^{2 n}}{(2 n-1) !} \int_{0}^{1}(1-t)^{2 n-1}\left(\frac{\partial}{\partial r}\right)^{k_{1}-j+2 n}(f)(r t, x) d t \\
& =-\frac{r^{2 n}}{(2 n-1) !} \int_{1}^{+\infty}(1-t)^{2 n-1}\left(\frac{\partial}{\partial r}\right)^{k_{1}-j+2 n}(f)(r t, x) d t \\
\left(\frac{\partial}{\partial x}\right)^{k_{2}-i}\left(\frac{\partial}{\partial r}\right)^{k_{1}-j} f(r, x) & =\frac{r^{2 n}}{(2 n-1) !} \int_{0}^{1}(1-t)^{2 n-1}\left(\frac{\partial}{\partial x}\right)^{k_{2}-i}\left(\frac{\partial}{\partial r}\right)^{k_{1}-j+2 n} f(r t, x) d t \\
& =-\frac{r^{2 n}}{(2 n-1) !} \int_{1}^{+\infty}(1-t)^{2 n-1}\left(\frac{\partial}{\partial x}\right)^{k_{2}-i}\left(\frac{\partial}{\partial r}\right)^{k_{1}-j+2 n} f(r t, x) d t
\end{aligned}
$$

The relations (4.9) and (4.11) imply that the function

$$
(r, x) \longmapsto\left(r^{2}+x^{2}\right)^{\gamma} f(r, x)
$$

belongs to $\mathcal{N}$ and that the mapping

$$
f \longmapsto\left(r^{2}+x^{2}\right)^{\gamma} f
$$

is continuous from $\mathcal{N}$ onto itself. The inverse mapping is given by

$$
f \longmapsto\left(r^{2}+x^{2}\right)^{-\gamma} f
$$

By the same way, we show that the mapping

$$
f \longmapsto|r|^{\gamma} f
$$

is an isomorphism from $\mathcal{N}$ onto itself.

(ii) Let $f \in \mathcal{N}$, and

$$
g(r, x)= \begin{cases}f\left(\sqrt{r^{2}-x^{2}}, x\right) & \text { if }|r| \geqslant|x| \\ 0 & \text { if }|r| \leqslant|x|\end{cases}
$$

we have

$$
\left(\frac{\partial}{\partial x}\right)^{k_{2}}\left(\frac{\partial}{\partial r}\right)^{k_{1}}(g)(r, x)=\sum_{j=0}^{k_{1}} P_{j}(r)\left(\sum_{p, q=0}^{k_{2}} Q_{p, q}(x)\left(\frac{\partial}{\partial x}\right)^{p}\left(\frac{\partial}{\partial r^{2}}\right)^{q+j}(f)\left(\sqrt{r^{2}-x^{2}}, x\right)\right),
$$

where $P_{j}$ and $Q_{p, q}$ are real polynomials. This equality, together with the fact that $f$ belongs to $\mathcal{N}$, implies that $g$ belongs to $\mathscr{S}_{*}\left(\mathbb{R}^{2}\right)$. 
14 Inversion formulas for Riemann-Liouville transform

Theorem 4.3. The Fourier transform $\mathfrak{F}_{\alpha}$ associated with Riemann-Liouville transform is an isomorphism from $\mathscr{Y}_{*}^{0}\left(\mathbb{R}^{2}\right)$ onto $\mathcal{N}$.

Proof. Let $f \in \mathscr{S}_{*}^{0}\left(\mathbb{R}^{2}\right)$. From the relation (3.12), we get

$$
\begin{aligned}
\left(\frac{\partial}{\partial \mu^{2}}\right)^{k} \mathfrak{F}_{\alpha}(f)(0, \lambda) & =\left(\frac{\partial}{\partial \mu^{2}}\right)^{k}\left(B \circ \widetilde{\mathfrak{F}}_{\alpha}(f)\right)(0, \lambda) \\
& =B\left(\left(\frac{\partial}{\partial \mu^{2}}\right)^{k} \widetilde{\mathfrak{F}}_{\alpha}(f)\right)(0, \lambda) \\
& =\left(\frac{\partial}{\partial \mu^{2}}\right)^{k} \widetilde{\mathfrak{F}}_{\alpha}(f)(\lambda, \lambda)=0,
\end{aligned}
$$

because $\operatorname{supp} \widetilde{\mathfrak{F}}_{\alpha}(f) \subset\left\{(\mu, \lambda) \in \mathbb{R}^{2},|\mu| \geqslant|\lambda|\right\}$, this shows that $\mathfrak{F}_{\alpha}$ maps injectively $\mathscr{S}_{*}^{0}\left(\mathbb{R}^{2}\right)$ into $\mathcal{N}$. On the other hand, let $h \in \mathcal{N}$ and

$$
g(r, x)= \begin{cases}h\left(\sqrt{r^{2}-x^{2}}, x\right) & \text { if }|r| \geqslant|x|, \\ 0 & \text { if }|r| \leqslant|x| .\end{cases}
$$

From Theorem 4.2(ii), $g$ belongs to $\mathscr{S}_{*}\left(\mathbb{R}^{2}\right)$, so there exists $f \in \mathscr{S}_{*}\left(\mathbb{R}^{2}\right)$ satisfying $\widetilde{\mathfrak{F}}_{\alpha}(f)=g$. Consequently, $f \in \mathscr{Y}_{*}^{0}\left(\mathbb{R}^{2}\right)$ and $\mathfrak{F}_{\alpha}(f)=h$.

From Lemmas 3.6, 4.1, and Theorem 4.3, we deduce the following result.

Corollary 4.4. The dual transform ${ }^{t} \Re_{\alpha}$ is an isomorphism from $\mathscr{S}_{*}^{0}\left(\mathbb{R}^{2}\right)$ onto $\mathscr{Y}_{*, 0}\left(\mathbb{R}^{2}\right)$.

\subsection{Inversion formula for $\mathfrak{R}_{\alpha}$ and ${ }^{t} \mathfrak{R}_{\alpha}$}

Theorem 4.5. (i) The operator $K_{\alpha}^{1}$ defined by

$$
K_{\alpha}^{1}(f)(r, x)=\Lambda_{\alpha}^{-1}\left(\frac{\pi}{2^{2 \alpha+1} \Gamma^{2}(\alpha+1)}\left(\mu^{2}+\lambda^{2}\right)^{\alpha}|\mu| \Lambda_{\alpha}(f)\right)(r, x)
$$

is an isomorphism from $\mathscr{S}_{*, 0}\left(\mathbb{R}^{2}\right)$ onto itself.

(ii) The operator $K_{\alpha}^{2}$ defined by

$$
K_{\alpha}^{2}(g)(r, x)=\mathfrak{F}_{\alpha}^{-1}\left(\frac{\pi}{2^{2 \alpha+1} \Gamma^{2}(\alpha+1)}\left(\mu^{2}+\lambda^{2}\right)^{\alpha}|\mu| \mathfrak{F}_{\alpha}(g)\right)(r, x)
$$

is an isomorphism from $\mathscr{Y}_{*}^{0}\left(\mathbb{R}^{2}\right)$ onto itself.

This theorem follows from Lemma 4.1, Theorems 4.2 and 4.3.

TheOREM 4.6. (i) For $f \in \mathscr{Y}_{*, 0}\left(\mathbb{R}^{2}\right)$ and $g \in \mathscr{S}_{*}^{0}\left(\mathbb{R}^{2}\right)$, there exists the inversion formula for $\mathfrak{R}_{\alpha}$ :

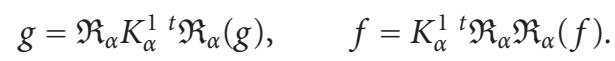

(ii) For $f \in \mathscr{Y}_{*, 0}\left(\mathbb{R}^{2}\right)$ and $g \in \mathscr{Y}_{*}^{0}\left(\mathbb{R}^{2}\right)$, there exists the inversion formula for ${ }^{t} \mathfrak{R}_{\alpha}$ :

$$
f={ }^{t} \mathfrak{R}_{\alpha} K_{\alpha}^{2} \Re_{\alpha}(f), \quad g=K_{\alpha}^{2} \Re_{\alpha}{ }^{t} \Re_{\alpha}(g) .
$$


Proof. (i) Let $g \in \mathscr{S}_{*}^{0}\left(\mathbb{R}^{2}\right)$. From the relation (2.15), Proposition 3.4, Lemma 3.6, and Theorem 4.3, we have

$$
\begin{aligned}
g(r, x) & =\int_{\mathbb{R}} \int_{0}^{+\infty}\left(\mu^{2}+\lambda^{2}\right)^{\alpha} \mu \Lambda_{\alpha} \circ{ }^{t} \mathfrak{R}_{\alpha}(g)(\mu, \lambda) \mathfrak{R}_{\alpha}(\cos (\mu .) \exp (i \lambda .))(r, x) d m(\mu, \lambda) \\
& =\mathfrak{R}_{\alpha}\left(\int_{\mathbb{R}} \int_{0}^{+\infty}\left(\mu^{2}+\lambda^{2}\right)^{\alpha} \mu \Lambda_{\alpha} \circ{ }^{t} \mathfrak{R}_{\alpha}(g)(\mu, \lambda) \cos (\mu .) \exp (i \lambda .) d m(\mu, \lambda)\right)(r, x) \\
& =\mathfrak{R}_{\alpha}\left(\Lambda_{\alpha}^{-1}\left(\frac{\pi}{2^{2 \alpha+1} \Gamma^{2}(\alpha+1)}\left(\mu^{2}+\lambda^{2}\right)^{\alpha}|\mu| \Lambda_{\alpha} \circ{ }^{t} \mathfrak{R}_{\alpha}(g)\right)\right)(r, x) \\
& =\mathfrak{R}_{\alpha} K_{\alpha}^{1}{ }^{t} \mathfrak{R}_{\alpha}(g)(r, x) .
\end{aligned}
$$

This relation, together with Corollary 4.4 and Theorem $4.5(\mathrm{i})$, implies that $\mathfrak{R}_{\alpha}$ is an isomorphism from $\mathscr{S}_{*, 0}\left(\mathbb{R}^{2}\right)$ onto $\mathscr{S}_{*}^{0}\left(\mathbb{R}^{2}\right)$, and that $K_{\alpha}^{1 t} \Re_{\alpha}$ is its inverse; in particular for $f \in \mathscr{S}_{*, 0}\left(\mathbb{R}^{2}\right)$, we have

$$
K_{\alpha}^{1 t} \Re_{\alpha} \Re_{\alpha}(f)=f
$$

(ii) Let $f \in \mathscr{S}_{*, 0}\left(\mathbb{R}^{2}\right)$. From (i), we have

$$
K_{\alpha}^{1 t} \Re_{\alpha} \Re_{\alpha}(f)=f .
$$

Let us put $g=\Re_{\alpha}(f)$, then $g \in \mathscr{Y}_{*}^{0}\left(\mathbb{R}^{2}\right)$, and we have

$$
\mathfrak{R}_{\alpha}^{-1}(g)=K_{\alpha}^{1 t} \mathfrak{R}_{\alpha}(g),
$$

and from Lemma 3.6, it follows that

$$
\begin{gathered}
\mathfrak{R}_{\alpha}^{-1}(g)=\Lambda_{\alpha}^{-1}\left(\frac{\pi}{2^{2 \alpha+1} \Gamma^{2}(\alpha+1)}\left(\mu^{2}+\lambda^{2}\right)^{\alpha}|\mu| \mathfrak{F}_{\alpha}(g)\right) \\
{ }^{t} \mathfrak{R}_{\alpha}^{-1} \mathfrak{R}_{\alpha}^{-1}(g)=\mathfrak{F}_{\alpha}^{-1}\left(\frac{\pi}{2^{2 \alpha+1} \Gamma^{2}(\alpha+1)}\left(\mu^{2}+\lambda^{2}\right)^{\alpha}|\mu| \mathfrak{F}_{\alpha}(g)\right)=K_{\alpha}^{2}(g),
\end{gathered}
$$

which gives

$$
f={ }^{t} \Re_{\alpha} K_{\alpha}^{2} \Re_{\alpha}(f)
$$

4.2. The expressions of the operators $K_{\alpha}^{1}$ and $K_{\alpha}^{2}$. In the previous subsection, we have defined the operators $K_{\alpha}^{1}$ and $K_{\alpha}^{2}$ in terms of Fourier transforms $\Lambda_{\alpha}$ and $\mathfrak{F}_{\alpha}$. Here, we will give nice expressions of these operators using fractional powers of partial differential operators. For this, we need the following inevitable notations.

(i) $\mathscr{E}_{*}(\mathbb{R})$ is the space of even infinitely differentiable functions on $\mathbb{R}$.

(ii) $\mathscr{S}_{*}(\mathbb{R})$ is the subspace of $\mathscr{E}_{*}(\mathbb{R})$, consisting of functions rapidly decreasing together with all their derivatives.

(iii) $\mathscr{S}_{*}^{\prime}(\mathbb{R})$ is the space of even tempered distributions on $\mathbb{R}$. 
16 Inversion formulas for Riemann-Liouville transform

(iv) $\mathscr{S}_{*}^{\prime}\left(\mathbb{R}^{2}\right)$ is the space of tempered distributions on $\mathbb{R}^{2}$, even with respect to the first variable.

Each of these spaces is equipped with its usual topology.

(i) For $a \in \mathbb{R}, a \geqslant-1 / 2, d \omega_{a}(r)$ is the measure defined on $[0,+\infty$ [ by

$$
d \omega_{a}(r)=\frac{1}{2^{a} \Gamma(a+1)} r^{2 a+1} d r
$$

(ii) $\ell_{a}$ is the Bessel operator defined on $] 0,+\infty[$ by

$$
\ell_{a}=\frac{d^{2}}{d r^{2}}+\frac{2 a+1}{r} \frac{d}{d r}, \quad a \geqslant-\frac{1}{2} .
$$

(iii) For an even measurable function $f$ on $\mathbb{R}, T_{f}^{\omega_{a}}$ is the element of $\mathscr{Y}_{*}^{\prime}(\mathbb{R})$, defined by

$$
\left\langle T_{f}^{\omega_{a}}, \varphi\right\rangle=\int_{0}^{+\infty} f(r) \varphi(r) d \omega_{a}(r), \quad \varphi \in \mathscr{I}_{*}(\mathbb{R}) .
$$

(iv) For a measurable function $g$ on $\mathbb{R}^{2}$, even with respect to the first variable, $T_{g}^{v}$ (resp., $\left.T_{g}^{m}\right)$ is the element of $\mathscr{Y}_{*}^{\prime}\left(\mathbb{R}^{2}\right)$, defined by

$$
\begin{gathered}
\left\langle T_{g}^{v}, \varphi\right\rangle=\int_{\mathbb{R}} \int_{0}^{+\infty} g(r, x) \varphi(r, x) d \nu(r, x), \\
\left(\text { resp., }\left\langle T_{g}^{m}, \varphi\right\rangle=\int_{\mathbb{R}} \int_{0}^{+\infty} g(r, x) \varphi(r, x) d m(r, x)\right), \quad \varphi \in \mathscr{Y}_{*}\left(\mathbb{R}^{2}\right),
\end{gathered}
$$

where $d v$ and $d m$ are the measures defined by the relations (3.1) and (3.26).

Definition 4.7. (i) The translation operator $\tau_{r}^{a}, r \in \mathbb{R}$, associated with Bessel operator $\ell_{a}$ is defined on $\mathscr{S}_{*}(\mathbb{R})$ by the following. For all $s \in \mathbb{R}$,

$$
\tau_{r}^{a} f(s)= \begin{cases}\frac{\Gamma(a+1)}{\sqrt{\pi} \Gamma(a+1 / 2)} \int_{0}^{\pi} f\left(\sqrt{r^{2}+s^{2}+2 r s \cos \theta}\right) \sin ^{2 a} \theta d \theta & \text { if } a>-\frac{1}{2} \\ \frac{f(r+s)+f(|r-s|)}{2} & \text { if } a=-\frac{1}{2}\end{cases}
$$

(ii) The convolution product of $f \in \mathscr{Y}_{*}(\mathbb{R})$ and $T \in \mathscr{Y}_{*}^{\prime}(\mathbb{R})$ is defined by

$$
\forall r \in \mathbb{R}, \quad T *_{a} f(r)=\left\langle T, \tau_{r}^{a} f\right\rangle .
$$

(iii) The Fourier Bessel transform is defined on $\mathscr{Y}_{*}(\mathbb{R})$ by

$$
\forall \mu \in \mathbb{R}, \quad F_{a}(f)(\mu)=\int_{0}^{+\infty} f(r) j_{a}(r \mu) d \omega_{a}(r),
$$

and on $\mathscr{Y}_{*}^{\prime}(\mathbb{R})$ by

$$
\forall \varphi \in \mathscr{I}_{*}(\mathbb{R}), \quad\left\langle F_{a}(T), \varphi\right\rangle=\left\langle T, F_{a}(\varphi)\right\rangle .
$$


We have the following properties (we refer to [19]).

(i) $F_{a}$ is an isomorphism from $\mathscr{Y}_{*}(\mathbb{R})\left(\operatorname{resp} ., \mathscr{S}_{*}^{\prime}(\mathbb{R})\right)$ onto itself, and we have

$$
F_{a}^{-1}=F_{a} .
$$

(ii) For $f \in \mathscr{Y}_{*}(\mathbb{R})$, and $r \in \mathbb{R}, \tau_{r}^{a} f$ belongs to $\mathscr{S}_{*}(\mathbb{R})$, and we have

$$
F_{a}\left(\tau_{r}^{a} f\right)(\mu)=j_{a}(r \mu) F_{a}(f)(\mu) .
$$

(iii) For $f \in \mathscr{Y}_{*}(\mathbb{R})$ and $T \in \mathscr{Y}_{*}^{\prime}(\mathbb{R})$, the function $T *_{a} f$ belongs to $\mathscr{E}_{*}(\mathbb{R})$, and is slowly increasing, moreover

$$
F_{a}\left(T_{T *_{a} f}^{\omega_{a}}\right)=F_{a}(f) F_{a}(T) .
$$

In the following, we will define the fractional powers of Bessel operator and the Laplacian operator defined on $\mathbb{R}^{2}$ by

$$
\Delta=\frac{\partial^{2}}{\partial r^{2}}+\frac{\partial^{2}}{\partial x^{2}}
$$

that we use to give simple expressions of $K_{\alpha}^{1}$ and $K_{\alpha}^{2}$.

In [16], the author has proved that the mappings

$$
z \longmapsto T_{|r|^{z}}^{\omega_{a}}, \quad z \longmapsto T_{\left(2^{z+a+1} \Gamma(z / 2+a+1) / \Gamma(-z / 2)\right)|r|^{-z-2 a-2}}^{\omega_{a}}
$$

defined initially for $-2(a+1)<\mathfrak{R} e(z)<0$, can be extended to a valued functions on $\mathscr{S}_{*}^{\prime}(\mathbb{R})$, analytic on $\mathbb{C} \backslash\left\{-2(k+a), k \in \mathbb{N}^{*}\right\}$, and we have

$$
T_{|r|^{z}}^{\omega_{a}}=F_{a}\left(T_{\left(2^{z+a+1} \Gamma(z / 2+a+1) / \Gamma(-z / 2)\right)|r|^{-z-2 a-2}}^{\omega_{a}}\right) .
$$

Definition 4.8. For $z \in \mathbb{C} \backslash\left\{-(k+a), k \in \mathbb{N}^{*}\right\}$, the fractional power of Bessel operator $\ell_{a}$ is defined on $\mathscr{Y}_{*}(\mathbb{R})$ by

$$
\left(-\ell_{a}\right)^{z} f(r)=\left(T_{\left(2^{2 z+a+1} \Gamma(z+a+1) / \Gamma(-z)\right)|s|^{-2 z-2 a-2}}^{\omega_{a}}\right) *_{a} f(r) .
$$

From the relations (4.40) and (4.43), we deduce that for $f \in \mathscr{I}_{*}(\mathbb{R})$ and $z \in \mathbb{C} \backslash\{-(k+$ a), $\left.k \in \mathbb{N}^{*}\right\}$, we have

$$
F_{a}\left(T_{\left(-\ell_{a}\right)^{z} f}^{\omega_{a}}\right)=F_{a}(f) T_{|r|^{2 z}}^{\omega_{a}} .
$$

On the other hand, from $[8,10]$, we deduce that the mappings

$$
z \longmapsto T_{\left(r^{2}+x^{2}\right)^{z}}^{m}, \quad T_{\sqrt{2 / \pi}\left(2^{2 z+\alpha+1} \Gamma(z+1) \Gamma(\alpha+1) / \Gamma(-z)\right)\left(r^{2}+x^{2}\right)^{-z-1}}^{m},
$$

defined initially for $-1<\mathfrak{R} e(z)<0$, can be extended to a valued functions in $\mathscr{Y}_{*}^{\prime}\left(\mathbb{R}^{2}\right)$, analytic on $\mathbb{C} \backslash\left\{-k, k \in \mathbb{N}^{*}\right\}$, and we have

$$
T_{\left(r^{2}+x^{2}\right)^{z}}^{m}=\Lambda_{\alpha}\left(T_{\sqrt{2 / \pi}\left(2^{2 z+\alpha+1} \Gamma(z+1) \Gamma(\alpha+1) / \Gamma(-z)\right)\left(r^{2}+x^{2}\right)^{-z-1}}^{m}\right),
$$


18 Inversion formulas for Riemann-Liouville transform

where $\Lambda_{\alpha}$ is defined on $\mathscr{Y}_{*}^{\prime}\left(\mathbb{R}^{2}\right)$ by

$$
\left\langle\Lambda_{\alpha}(T), \varphi\right\rangle=\left\langle T, \Lambda_{\alpha}(\varphi)\right\rangle, \quad \varphi \in \mathscr{I}_{*}\left(\mathbb{R}^{2}\right),
$$

and $\Lambda_{\alpha}(\varphi)$ is given in Lemma 3.6.

Definition 4.9. For $z \in \mathbb{C} \backslash\left\{-k, k \in \mathbb{N}^{*}\right\}$, the fractional power of the Laplacian operator $\Delta$ is defined on $\mathscr{Y}_{*}\left(\mathbb{R}^{2}\right)$ by

$$
(-\Delta)^{z} f(r, x)=\left(T_{(1 / \pi)\left(2^{2 z+1} \Gamma(z+1) / \Gamma(-z)\right)\left(s^{2}+y^{2}\right)^{-z-1} * f}^{m}\right)(r, x),
$$

where

(i) $*$ is the usual convolution product defined by

$$
T * f(r, x)=\left\langle T, \sigma_{(r, x)} \check{f}\right\rangle, \quad T \in \mathscr{Y}_{*}^{\prime}\left(\mathbb{R}^{2}\right), f \in \mathscr{Y}_{*}\left(\mathbb{R}^{2}\right)
$$

(ii)

$$
\sigma_{(r, x)} f(s, y)=\frac{1}{2}[f(r+s, y-x)+f(r-s, y-x)], \quad f \in \mathscr{Y}_{*}\left(\mathbb{R}^{2}\right) .
$$

It is well known that for $f \in \mathscr{Y}_{*}\left(\mathbb{R}^{2}\right)$ and $T \in \mathscr{Y}_{*}^{\prime}\left(\mathbb{R}^{2}\right)$, the function $T * f$ belongs to $\mathscr{E}_{*}\left(\mathbb{R}^{2}\right)$ and is slowly increasing, and we have

$$
\Lambda_{\alpha}\left(T_{T * f}^{m}\right)=\Lambda_{\alpha}(f) \Lambda_{\alpha}(T),
$$

thus from the relations (4.47) and (4.52), we deduce that for $f \in \mathscr{I}_{*}\left(\mathbb{R}^{2}\right)$ and $z \in \mathbb{C} \backslash$ $\left\{-k, k \in \mathbb{N}^{*}\right\}$,

$$
\Lambda_{\alpha}\left(T_{\sqrt{2 \pi} 2^{\alpha} \Gamma(\alpha+1)(-\Delta)^{z} f}^{m}\right)=\Lambda_{\alpha}(f) T_{\left(r^{2}+x^{2}\right)^{z}}^{m}
$$

Theorem 4.10. The operator $K_{\alpha}^{1}$ defined in Theorem 4.5 can be written as

$$
K_{\alpha}^{1}(f)=\frac{\pi}{2^{2 \alpha+1} \Gamma^{2}(\alpha+1)}\left(-\frac{\partial^{2}}{\partial r^{2}}\right)^{1 / 2}(-\Delta)^{\alpha} f,
$$

where

$$
\left(-\frac{\partial^{2}}{\partial r^{2}}\right)^{1 / 2} f(r, x)=\left(-\ell_{-1 / 2}\right)^{1 / 2}(f(\cdot, x))(r) .
$$

Proof. Let $f \in \mathscr{Y}_{*, 0}\left(\mathbb{R}^{2}\right)$. Using Fubini's theorem, we get for every $\varphi \in \mathscr{I}_{*}\left(\mathbb{R}^{2}\right)$ the following:

$$
\begin{aligned}
& \left\langle\Lambda _ { \alpha } \left( T_{\left.\left.\left(-\partial^{2} / \partial r^{2}\right)^{1 / 2} f\right), \varphi\right\rangle}^{m}\right.\right. \\
& \quad=\frac{1}{2^{2 \alpha+2} \Gamma^{2}(\alpha+1)} \int_{\mathbb{R}} \int_{0}^{+\infty}\left\langle T_{\left(-\ell_{-1 / 2}\right)^{1 / 2}(f(\cdot, x))}^{\omega_{-1 / 2}}, F_{-1 / 2}(\varphi(\cdot, y))\right\rangle \times \exp (-i x y) d x d y
\end{aligned}
$$


and by the relation (4.45), we obtain

$$
\begin{aligned}
& \left\langle\Lambda _ { \alpha } \left( T_{\left.\left.\left(-\partial^{2} / \partial r^{2}\right)^{1 / 2} f\right), \varphi\right\rangle}^{m}\right.\right. \\
& \quad=\frac{1}{2^{2 \alpha+2} \Gamma^{2}(\alpha+1)} \int_{\mathbb{R}} \int_{0}^{+\infty}\left\langle F_{-1 / 2}(f(\cdot, x)) T_{|r|}^{\omega_{-1 / 2}}, \varphi(\cdot, y)\right\rangle \times \exp (-i x y) d x d y,
\end{aligned}
$$

which involves that

$$
\left\langle\Lambda_{\alpha}\left(T_{\left(-\partial^{2} / \partial r^{2}\right)^{1 / 2} f}^{m}\right), \varphi\right\rangle=\int_{\mathbb{R}} \int_{0}^{+\infty} r \Lambda_{\alpha}(f)(r, y) \varphi(r, y) d m(r, y),
$$

this shows that

$$
\Lambda_{\alpha}\left(T_{\left(-\partial^{2} / \partial r^{2}\right)^{1 / 2} f}^{m}\right)=T_{|r| \Lambda_{\alpha}(f)}^{m} .
$$

Now, from Lemma 4.1, we deduce that the function

$$
(\mu, \lambda) \longmapsto|\mu| \Lambda_{\alpha}(f)(\mu, \lambda)
$$

belongs to the subspace $\mathcal{N}$. Then, from the relation (4.59), it follows that the function $\left(-\partial^{2} / \partial r^{2}\right)^{1 / 2} f$ belongs to the subspace $\mathscr{S}_{*, 0}\left(\mathbb{R}^{2}\right)$, and we have

$$
\forall(\mu, \lambda) \in \mathbb{R}^{2}, \quad \Lambda_{\alpha}\left(\left(-\frac{\partial^{2}}{\partial r^{2}}\right)^{1 / 2} f\right)(\mu, \lambda)=|\mu| \Lambda_{\alpha}(\mu, \lambda)
$$

By the same way, and using the relation (4.53), we deduce that for every $f \in \mathscr{S}_{*, 0}\left(\mathbb{R}^{2}\right)$, the function $(-\Delta)^{\alpha} f$ belongs to the subspace $\mathscr{S}_{*, 0}\left(\mathbb{R}^{2}\right)$, and we have that for all $(\mu, \lambda) \in \mathbb{R}^{2}$,

$$
\Lambda_{\alpha}\left(\sqrt{2 \pi} 2^{\alpha} \Gamma(\alpha+1)(-\Delta)^{\alpha} f\right)(\mu, \lambda)=\left(\mu^{2}+\lambda^{2}\right)^{\alpha} \Lambda_{\alpha}(f)(\mu, \lambda) .
$$

Hence, the theorem follows from the relations (4.61) and (4.62).

Definition 4.11. Let $a, b \in \mathbb{R}, b \geqslant a \geqslant-1 / 2$.

(i) The Sonine transform is the mapping defined on $\mathscr{E}_{*}(\mathbb{R})$ by the following. For all $r \in \mathbb{R}$,

$$
S_{b, a}(f)(r)= \begin{cases}\frac{2 \Gamma(b+1)}{\Gamma(b-a) \Gamma(a+1)} \int_{0}^{1}\left(1-t^{2}\right)^{b-a-1} f(r t) t^{2 a+1} d t & \text { if } b>a, \\ f(r) & \text { if } b=a .\end{cases}
$$

(ii) The dual transform ${ }^{t} S_{b, a}$ is the mapping defined on $\mathscr{S}_{*}(\mathbb{R})$ by the following. For all $r \in \mathbb{R}$,

$$
{ }^{t} S_{b, a}(f)(r)= \begin{cases}\frac{2 \Gamma(b+1)}{\Gamma(b-a) \Gamma(a+1)} \int_{r}^{+\infty}\left(t^{2}-r^{2}\right)^{b-a-1} f(t) t d t & \text { if } b>a, \\ f(r) & \text { if } b=a .\end{cases}
$$


20 Inversion formulas for Riemann-Liouville transform

Then, we have the following.

(i) The Sonine transform is an isomorphism from $\mathscr{E}_{*}(\mathbb{R})$ onto itself.

(ii) The dual Sonine transform is an isomorphism from $\mathscr{S}_{*}(\mathbb{R})$ onto itself.

(iii) For $f \in \mathscr{E}_{*}(\mathbb{R}), f$ bounded, and $g \in \mathscr{Y}_{*}(\mathbb{R})$, we have

$$
\int_{0}^{+\infty} S_{b, a}(f)(r) g(r) r^{2 b+1} d r=\int_{o}^{+\infty} f(r)^{t} S_{b, a}(g)(r) r^{2 a+1} d r
$$

(iv) $j_{b}=S_{b, a}\left(j_{a}\right)$.

(v)

$$
F_{b}=\frac{\Gamma(a+1)}{2^{b-a} \Gamma(b+1)} F_{a} \circ{ }^{t} S_{b, a} .
$$

For more details, we refer to $[18,20,21]$.

We denote the following.

(i) For $T \in \mathscr{Y}_{*}^{\prime}\left(\mathbb{R}^{2}\right), \varphi \in \mathscr{S}_{*}\left(\mathbb{R}^{2}\right)$,

$$
\left\langle S_{a, 0}(T), \varphi\right\rangle=\langle T, \psi\rangle,
$$

with $\psi(r, x)={ }^{t} S_{a, 0}(\varphi(\cdot, x))(r)$.

(ii) For all $(r, x) \in \mathbb{R}^{2}$,

$$
T \# \varphi(r, x)=\left\langle T, \mathscr{T}_{(r,-x)} \check{\varphi}\right\rangle,
$$

where $\mathscr{T}_{(r, x)}$ is the translation operator given by Definition 3.1.

(iii) $\widetilde{\mathfrak{F}}_{\alpha}$ is the mapping defined on $\mathscr{S}_{*}^{\prime}\left(\mathbb{R}^{2}\right)$ by

$$
\forall \varphi \in \mathscr{S}_{*}\left(\mathbb{R}^{2}\right), \quad\left\langle\widetilde{\mathfrak{F}}_{\alpha}(T), \varphi\right\rangle=\left\langle T, \widetilde{\mathfrak{F}}_{\alpha}(\varphi)\right\rangle .
$$

(iv) $L_{\alpha}$ is the operator defined on $\mathscr{Y}_{*}\left(\mathbb{R}^{2}\right)$ by

$$
L_{\alpha} f(r, x)=\left(-\ell_{\alpha}\right)^{2 \alpha}(f(\cdot, x))(r)
$$

where $\left(-\ell_{\alpha}\right)^{z}$ is the fractional power of Bessel given by Definition 4.8.

Theorem 4.12. The operator $K_{\alpha}^{2}$, defined in Theorem 4.5, is given by

$$
K_{\alpha}^{2}(f)(r, x)=\frac{\pi}{2^{4 \alpha+2} \Gamma^{4}(\alpha+1)} S_{\alpha, 0}(T) \#\left(-\Delta_{2}\right) L_{\alpha}(\check{f})(r,-x), \quad f \in \mathscr{S}_{*}^{0}\left(\mathbb{R}^{2}\right),
$$

where

(i) $T$ is the distribution defined by

$$
\langle T, \varphi\rangle=\int_{\mathbb{R}} \varphi(y, y) d y
$$

(ii) $\Delta_{2}$ is the operator defined in Section 2. 
Proof. By the definition of $K_{\alpha}^{2}$, and the relation (3.12), we have that for $f \in \mathscr{S}_{*}^{0}\left(\mathbb{R}^{2}\right)$,

$$
\begin{aligned}
& K_{\alpha}^{2}(f)(r, x) \\
& \quad=\frac{\sqrt{\pi / 2}}{2^{3 \alpha+1} \Gamma^{3}(\alpha+1)} \int_{\mathbb{R}} \int_{0}^{+\infty} \mu^{2}\left(\mu^{2}+\lambda^{2}\right)^{2 \alpha} \widetilde{\mathfrak{F}}_{\alpha}(f)\left(\sqrt{\mu^{2}+\lambda^{2}}, \lambda\right) j_{\alpha}\left(r \sqrt{\mu^{2}+\lambda^{2}}\right) \exp (i \lambda x) d \mu d \lambda .
\end{aligned}
$$

By a change of variables, and using Fubini's theorem, we get

$$
K_{\alpha}^{2}(f)(r, x)=\frac{\sqrt{\pi / 2}}{2^{3 \alpha+1} \Gamma^{3}(\alpha+1)} \int_{0}^{+\infty} \int_{-\nu}^{\nu} \nu^{4 \alpha}\left(\nu^{2}-\lambda^{2}\right) \widetilde{\mathfrak{F}}_{\alpha}(f)(\nu, \lambda) \frac{\exp (i \lambda x)}{\sqrt{\nu^{2}-\lambda^{2}}} j_{\alpha}(r \nu) \nu d \nu d \lambda .
$$

On the other hand, for $f \in \mathscr{S}_{*}^{0}\left(\mathbb{R}^{2}\right)$, the function $L_{\alpha} f$ belongs to $\mathscr{E}_{*}\left(\mathbb{R}^{2}\right)$, and is slowly increasing. Moreover, we have

$$
\widetilde{\mathfrak{F}}_{\alpha}\left(T_{L_{\alpha} f}^{\nu}\right)=T_{|\mu|+\alpha}^{\nu} \widetilde{\mathfrak{F}}_{\alpha}(f)
$$

But, for $f \in \mathscr{Y}_{*}^{0}\left(\mathbb{R}^{2}\right)$, the function $\widetilde{\mathfrak{F}}_{\alpha}(f)$ belongs to the subspace $\mathcal{N}$; according to Theorem 4.2, we deduce that the function $L_{\alpha} f$ belongs to $\mathscr{S}_{*}\left(\mathbb{R}^{2}\right)$, and we have

$$
\forall(\mu, \lambda) \in \mathbb{R}^{2}, \quad \widetilde{\mathfrak{F}}_{\alpha}\left(L_{\alpha} f\right)(\mu, \lambda)=|\mu|^{4 \alpha} \widetilde{\mathfrak{F}}_{\alpha}(f)(\mu, \lambda) .
$$

This involves that

$$
\begin{aligned}
K_{\alpha}^{2}(f)(r, x) & =\frac{\sqrt{\pi / 2}}{2^{3 \alpha+1} \Gamma^{3}(\alpha+1)} \int_{0}^{+\infty} \int_{-\nu}^{\nu}\left(\nu^{2}-\lambda^{2}\right) \widetilde{\mathfrak{F}}_{\alpha}\left(L_{\alpha} f\right)(\nu, \lambda) \frac{\exp (i \lambda x)}{\sqrt{\nu^{2}-\lambda^{2}}} j_{\alpha}(r \nu) \nu d \nu d \lambda \\
& =\frac{\sqrt{\pi / 2}}{2^{3 \alpha+1} \Gamma^{3}(\alpha+1)} \int_{0}^{+\infty} \int_{-\nu}^{\nu} \widetilde{\mathfrak{F}}_{\alpha}\left(\left(-\Delta_{2}\right) L_{\alpha} f\right)(\nu, \lambda) \frac{\exp (i \lambda x)}{\sqrt{\nu^{2}-\lambda^{2}}} j_{\alpha}(r \nu) \nu d \nu d \lambda
\end{aligned}
$$

Since for every $f \in \mathscr{S}_{*}\left(\mathbb{R}^{2}\right)$, we have that

$$
\forall(r, x),(\mu, \lambda) \in \mathbb{R}^{2}, \quad \widetilde{\mathfrak{F}}_{\alpha}\left(\mathcal{T}_{(r, x)} f\right)(\nu, \lambda)=j_{\alpha}(r \nu) \exp (i \lambda x) \widetilde{\mathfrak{F}}_{\alpha}(f)(\nu, \lambda),
$$

we get

$$
K_{\alpha}^{2}(f)(r, x)=\frac{\sqrt{\pi / 2}}{2^{3 \alpha+1} \Gamma^{3}(\alpha+1)} \int_{0}^{+\infty} \int_{-v}^{\nu} \widetilde{\mathfrak{F}}_{\alpha}\left(\mathscr{T}_{(r, x)}\left(-\Delta_{2}\right) L_{\alpha} f\right)(\nu, \lambda) \frac{\nu d \nu d \lambda}{\sqrt{\nu^{2}-\lambda^{2}}} .
$$

Using the expression of $\widetilde{\mathfrak{F}}_{\alpha}$, we obtain

$$
\begin{aligned}
K_{\alpha}^{2}(f)(r, x)=\frac{1}{2^{4 \alpha+2} \Gamma^{4}(\alpha+1)} \int_{0}^{+\infty} \int_{-\nu}^{\nu}\left[\int_{\mathbb{R}} \int_{0}^{+\infty}\right. & \left(\mathcal{T}_{(r, x)}\left(-\Delta_{2}\right) L_{\alpha} f\right)(s, y) \\
& \left.\times j_{\alpha}(s \nu) \exp (-i \lambda y) s^{2 \alpha+1} d s d y\right] \frac{d \lambda}{\sqrt{\nu^{2}-\lambda^{2}}} \nu d \nu .
\end{aligned}
$$


22 Inversion formulas for Riemann-Liouville transform

From the fact that

$$
\int_{-v}^{v} \frac{\exp (-i \lambda y)}{\sqrt{v^{2}-\lambda^{2}}} d \lambda=\pi j_{0}(\nu y)
$$

and using Fubini's theorem, we deduce that

$$
\begin{aligned}
K_{\alpha}^{2}(f) & (r, x) \\
= & \frac{\pi}{2^{4 \alpha+2} \Gamma^{4}(\alpha+1)} \int_{\mathbb{R}}\left\{\iint_{0}^{+\infty}\left(\mathcal{T}_{(r, x)}\left(-\Delta_{2}\right) L_{\alpha} f\right)(s, y) j_{\alpha}(s \nu) \times j_{0}(\nu y) s^{2 \alpha+1} d s \nu d \nu\right\} d y \\
\quad= & \frac{\pi}{2^{3 \alpha+2} \Gamma^{3}(\alpha+1)} \int_{\mathbb{R}}\left\{\int_{0}^{+\infty} F_{\alpha}\left(\left(\mathcal{T}_{(r, x)}\left(-\Delta_{2}\right) L_{\alpha} f\right)(\cdot, y)\right)(\nu) j_{0}(\nu y) \nu d \nu\right\} d y,
\end{aligned}
$$

and from the relation (4.66), we have

$$
\begin{aligned}
& K_{\alpha}^{2}(f)(r, x) \\
& \quad=\frac{\pi}{2^{4 \alpha+2} \Gamma^{4}(\alpha+1)} \int_{\mathbb{R}}\left\{\int_{0}^{+\infty} F_{0} \circ{ }^{t} S_{\alpha, 0}\left(\left(\mathscr{T}_{(r, x)}\left(-\Delta_{2}\right) L_{\alpha} f\right)(\cdot, y)\right)(\nu) \times j_{0}(\nu y) \nu d \nu\right\} d y,
\end{aligned}
$$

and the relation (4.38) implies that

$$
K_{\alpha}^{2}(f)(r, x)=\frac{\pi}{2^{4 \alpha+2} \Gamma^{4}(\alpha+1)} \int_{\mathbb{R}}{ }^{t} S_{\alpha, 0}\left(\left(\mathscr{T}_{(r, x)}\left(-\Delta_{2}\right) L_{\alpha} f\right)(\cdot, y)\right)(y) d y .
$$

\subsection{Plancherel theorem for ${ }^{t} \Re_{\alpha}$}

Proposition 4.13. The operator $K_{\alpha}^{3}$ defined by

$$
K_{\alpha}^{3}(f)=\pi\left(-\frac{\partial^{2}}{\partial r^{2}}\right)^{1 / 4}(-\triangle)^{\alpha / 2} f
$$

is an isomorphism from $\mathscr{Y}_{*, 0}\left(\mathbb{R}^{2}\right)$ onto itself, where

$$
\left(-\frac{\partial^{2}}{\partial r^{2}}\right)^{1 / 4} f(r, x)=\left(-\ell_{-1 / 2}\right)^{1 / 4}(f(\cdot, x))(r)
$$

Proof. Let $f \in \mathscr{I}_{*, 0}\left(\mathbb{R}^{2}\right)$. From the relations (4.45) and (4.53), we deduce that for all $(\mu, \lambda)$ $\in \mathbb{R}^{2}$,

$$
\sqrt{|\mu|}\left(\mu^{2}+\lambda^{2}\right)^{\alpha / 2} \Lambda_{\alpha}(f)(\mu, \lambda)=\Lambda_{\alpha}\left(\sqrt{2 \pi} 2^{\alpha} \Gamma(\alpha+1)\left(-\frac{\partial^{2}}{\partial r^{2}}\right)^{1 / 4}(-\Delta)^{\alpha / 2} f\right)(\mu, \lambda)
$$


which implies that for all $(\mu, \lambda) \in \mathbb{R}^{2}$,

$$
\Lambda_{\alpha}\left(K_{\alpha}^{3}\right)(f)(\mu, \lambda)=\sqrt{\frac{\pi}{2}} \frac{1}{2^{\alpha} \Gamma(\alpha+1)} \sqrt{|\mu|}\left(\mu^{2}+\lambda^{2}\right)^{\alpha / 2} \Lambda_{\alpha}(f)(\mu, \lambda) .
$$

Then, the result follows from Lemma 4.1 and Theorem 4.2.

Proposition 4.14. For $g \in \mathscr{Y}_{*}^{0}\left(\mathbb{R}^{2}\right)$, there exists the Plancherel formula

$$
\int_{\mathbb{R}} \int_{0}^{+\infty}|g(r, x)|^{2} d \nu(r, x)=\int_{\mathbb{R}} \int_{0}^{+\infty}\left|K_{\alpha}^{3}\left({ }^{t} \Re_{\alpha}(g)\right)(r, x)\right|^{2} d m(r, x) .
$$

Proof. Let $g \in \mathscr{Y}_{*}^{0}\left(\mathbb{R}^{2}\right)$, from Theorem 3.5 (Plancherel formula), we have

$$
\int_{\mathbb{R}} \int_{0}^{+\infty}|g(r, x)|^{2} d \nu(r, x)=\iint_{\Gamma}\left|\mathfrak{F}_{\alpha}(g)(\mu, \lambda)\right|^{2} d \gamma(\mu, \lambda) .
$$

From the relation (3.12), Lemma 3.6, and the fact that

$$
\operatorname{supp} \widetilde{\mathfrak{F}}_{\alpha}(g) \subset\left\{(\mu, \lambda) \in \mathbb{R}^{2} /|\mu| \geqslant|\lambda|\right\}
$$

we get

$$
\int_{\mathbb{R}} \int_{0}^{+\infty}|g(r, x)|^{2} d \nu(r, x)=\int_{\mathbb{R}} \int_{0}^{+\infty}\left|\sqrt{\mu}\left(\mu^{2}+\lambda^{2}\right)^{\alpha / 2} \Lambda_{\alpha} \circ{ }^{t} \Re_{\alpha}(g)(\mu, \lambda)\right|^{2} d m(\mu, \lambda) .
$$

We complete the proof by using the formula (4.88), and the fact that for every $f \in$ $\mathscr{S}_{*}\left(\mathbb{R}^{2}\right)$

$$
\int_{\mathbb{R}} \int_{0}^{+\infty}\left|\Lambda_{\alpha}(f)(\mu, \lambda)\right|^{2} d m(\mu, \lambda)=\frac{\pi}{2^{2 \alpha+1} \Gamma^{2}(\alpha+1)} \int_{\mathbb{R}} \int_{0}^{+\infty}|f(\mu, \lambda)|^{2} d m(\mu, \lambda) .
$$

We denote by

(i) $L_{0}^{2}(d v)$ the subspace of $L^{2}(d v)$ consisting of functions $g$ such that

$$
\operatorname{supp} \widetilde{\mathfrak{F}}_{\alpha}(g) \subset\left\{(\mu, \lambda) \in \mathbb{R}^{2} /|\mu| \geqslant|\lambda|\right\}
$$

(ii) $L^{2}(d m)$ the space of square integrable functions on $[0,+\infty[\times \mathbb{R}$ with respect to the measure $d m(r, x)$.

THeOREM 4.15. The operator $K_{\alpha}^{3} \circ{ }^{t} \mathfrak{R}_{\alpha}$ can be extended to an isometric isomorphism from $L_{0}^{2}(d v)$ onto $L^{2}(d m)$. 
24 Inversion formulas for Riemann-Liouville transform

Proof. The theorem follows from Propositions 4.13, 4.14, and the density of $\mathscr{Y}_{*, 0}\left(\mathbb{R}^{2}\right)$ (resp., $\left.\mathscr{S}_{*}^{0}\left(\mathbb{R}^{2}\right)\right)$ in $L^{2}(d m)$ (resp., $\left.L_{0}^{2}(d v)\right)$.

\section{Transmutation operators}

Proposition 5.1. The Riemann-Liouville transform and its dual satisfy the following permutation properties.

(i) For all $f \in \mathscr{Y}_{*}\left(\mathbb{R}^{2}\right)$,

$$
{ }^{t} \mathfrak{R}_{\alpha}\left(\Delta_{2} f\right)=\frac{\partial^{2}}{\partial r^{2}}{ }^{t} \mathfrak{R}_{\alpha}(f), \quad{ }^{t} \mathfrak{R}_{\alpha}\left(\Delta_{1} f\right)=\Delta_{1}{ }^{t} \mathfrak{R}_{\alpha}(f) .
$$

(ii) For all $f \in \mathscr{E}_{*}\left(\mathbb{R}^{2}\right)$,

$$
\Delta_{2} \mathfrak{R}_{\alpha}(f)=\mathfrak{R}_{\alpha}\left(\frac{\partial^{2} f}{\partial r^{2}}\right), \quad \Delta_{1} \mathfrak{R}_{\alpha}(f)=\mathfrak{R}_{\alpha}\left(\Delta_{1} f\right)
$$

Proof. (i) We know that the operators $\Delta_{1}, \Delta_{2}, \partial^{2} / \partial r^{2}$, and ${ }^{t} \Re_{\alpha}$ are continuous mappings from $\mathscr{S}_{*}\left(\mathbb{R}^{2}\right)$ into itself. Then, by applying the usual Fourier transform $\Lambda_{\alpha}$, we have

$$
\begin{gathered}
\Lambda_{\alpha}\left({ }^{t} \mathfrak{R}_{\alpha}\left(\Delta_{2} f\right)\right)(\mu, \lambda)=-\mu^{2} \Lambda_{\alpha}{ }^{t}{ }^{t} \mathfrak{R}_{\alpha}(f)(\mu, \lambda)=\Lambda_{\alpha}\left(\frac{\partial^{2}}{\partial r^{2}}{ }^{t} \mathfrak{R}_{\alpha}(f)\right)(\mu, \lambda), \\
\Lambda_{\alpha}\left(\Delta_{1}{ }^{t} \mathfrak{R}_{\alpha} f\right)(\mu, \lambda)=i \lambda \Lambda_{\alpha}\left({ }^{t} \mathfrak{R}_{\alpha}(f)\right)(\mu, \lambda)=\Lambda_{\alpha}\left({ }^{t} \mathfrak{R}_{\alpha}\left(\Delta_{1} f\right)\right)(\mu, \lambda) .
\end{gathered}
$$

Consequently, (i) follows from the fact that $\Lambda_{\alpha}$ is an isomorphism from $\mathscr{S}_{*}\left(\mathbb{R}^{2}\right)$ onto itself.

(ii) We obtain the result from (i), Lemma 2.4 , and the fact that for $f \in \mathscr{E}_{*}\left(\mathbb{R}^{2}\right)$, and $g \in \mathscr{D}_{*}\left(\mathbb{R}^{2}\right)$,

$$
\int_{\mathbb{R}} \int_{0}^{+\infty} \Delta_{2} f(r, x) g(r, x) d \nu(r, x)=\int_{\mathbb{R}} \int_{0}^{+\infty} f(r, x) \Delta_{2} g(r, x) d \nu(r, x) .
$$

TheOREM 5.2. (i) The Riemann-Liouville transform $\mathfrak{R}_{\alpha}$ is a transmutation operator of

$$
\frac{\partial^{2}}{\partial r^{2}}, \Delta_{1} \quad \text { into } \Delta_{2}, \Delta_{1}
$$

from

$$
\mathscr{S}_{*, 0}\left(\mathbb{R}^{2}\right) \text { onto } \mathscr{Y}_{*}^{0}\left(\mathbb{R}^{2}\right) .
$$


(ii) The dual transform ${ }^{t} \mathfrak{R}_{\alpha}$ is a transmutation operator of

$$
\Delta_{2}, \Delta_{1} \quad \text { into } \frac{\partial^{2}}{\partial r^{2}}, \Delta_{1}
$$

from

$$
\mathscr{S}_{*}^{0}\left(\mathbb{R}^{2}\right) \text { onto } \mathscr{Y}_{*, 0}\left(\mathbb{R}^{2}\right)
$$

This theorem follows from Proposition 5.1 and the fact that $\mathfrak{R}_{\alpha}$ is an isomorphism from $\mathscr{S}_{*, 0}\left(\mathbb{R}^{2}\right)$ onto $\mathscr{S}_{*}^{0}\left(\mathbb{R}^{2}\right)$ and ${ }^{t} \mathfrak{R}_{\alpha}$ is an isomorphism from $\mathscr{S}_{*}^{0}\left(\mathbb{R}^{2}\right)$ onto $\mathscr{Y}_{*, 0}\left(\mathbb{R}^{2}\right)$.

\section{References}

[1] L.-E. Andersson, On the determination of a function from spherical averages, SIAM Journal on Mathematical Analysis 19 (1988), no. 1, 214-232.

[2] R. Courant and D. Hilbert, Methods of Mathematical Physics. Vol. II: Partial Differential Equations, Wiley-Interscience, New York, 1962.

[3] L. Debnath, Fractional integral and fractional differential equations in fluid mechanics, Fractional Calculus \& Applied Analysis 6 (2003), no. 2, 119-155.

[4] _ Recent applications of fractional calculus to science and engineering, International Journal of Mathematics and Mathematical Sciences 2003 (2003), no. 54, 3413-3442.

[5] L. Debnath and D. D. Bhatta, Solutions to few linear fractional inhomogeneous partial differential equations in fluid mechanics, Fractional Calculus \& Applied Analysis 7 (2004), no. 1, 21-36.

[6] J. A. Fawcett, Inversion of n-dimensional spherical averages, SIAM Journal on Applied Mathematics 45 (1985), no. 2, 336-341.

[7] T. G. Genchev, Entire functions of exponential type with polynomial growth on $\mathbb{R}_{x}^{n}$, Journal of Mathematical Analysis and Applications 60 (1977), no. 1, 103-119.

[8] F. B. Gonzalez, Radon transforms on Grassmann manifolds, Journal of Functional Analysis 71 (1987), no. 2, 339-362.

[9] J. Gosselin and K. Stempak, A weak-type estimate for Fourier-Bessel multipliers, Proceedings of the American Mathematical Society 106 (1989), no. 3, 655-662.

[10] S. Helgason, The Radon Transform, Progress in Mathematics, vol. 5, Birkhäuser, Massachusetts, 1980.

[11] H. Hellsten and L.-E. Andersson, An inverse method for the processing of synthetic aperture radar data, Inverse Problems 3 (1987), no. 1, 111-124.

[12] M. Herberthson, A numerical implementation of an inverse formula for CARABAS raw data, Internal Report D 30430-3.2, National Defense Research Institute, FOA, Box 1165; S-581 11, Linköping, 1986.

[13] N. N. Lebedev, Special Functions and Their Applications, Dover, New York, 1972.

[14] D. Ludwig, The Radon transform on Euclidean space, Communications on Pure and Applied Mathematics 19 (1966), 49-81.

[15] M. M. Nessibi, L. T. Rachdi, and K. Trimeche, Ranges and inversion formulas for spherical mean operator and its dual, Journal of Mathematical Analysis and Applications 196 (1995), no. 3, 861884.

[16] L. T. Rachdi, Fractional powers of Bessel operator and inversion formulas for Riemann-Liouville and Weyl transforms, Journal of Mathematical Sciences 12 (2001), no. 1.

[17] D. C. Solmon, Asymptotic formulas for the dual Radon transform and applications, Mathematische Zeitschrift 195 (1987), no. 3, 321-343.

[18] K. Trimèche, Transformation intégrale de Weyl et théorème de Paley-Wiener associés à un opérateur différentiel singulier sur $(0, \infty)$ [Weyl integral transform and Paley-Wiener theorem associated 


\section{Inversion formulas for Riemann-Liouville transform}

with a singular differential operator on $(0, \infty)$ ], Journal de Mathématiques Pures et Appliquées. Neuvième Série 60 (1981), no. 1, 51-98.

[19] _ Inversion of the Lions transmutation operators using generalized wavelets, Applied and Computational Harmonic Analysis 4 (1997), no. 1, 97-112.

[20] Generalized Harmonic Analysis and Wavelet Packets: An Elementary Treatment of Theory $\&$ Applications, Gordon and Breach Science, Amsterdam, 2001.

[21] G. N. Watson, A Treatise on the Theory of Bessel Functions, 2nd ed., Cambridge University Press, London, 1966.

C. Baccar: Department of Mathematics, Faculty of Sciences of Tunis, University Tunis El Manar, 2092 Tunis, Tunisia

N. B. Hamadi: Department of Mathematics, Faculty of Sciences of Tunis, University Tunis El Manar, 2092 Tunis, Tunisia

L. T. Rachdi: Department of Mathematics, Faculty of Sciences of Tunis, University Tunis El Manar, 2092 Tunis, Tunisia

E-mail address: lakhdartannech.rachdi@fst.rnu.tn 


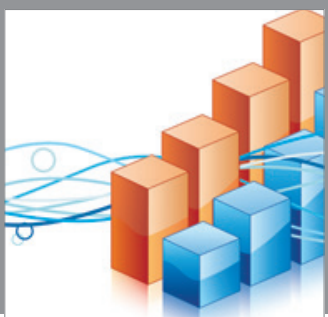

Advances in

Operations Research

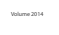

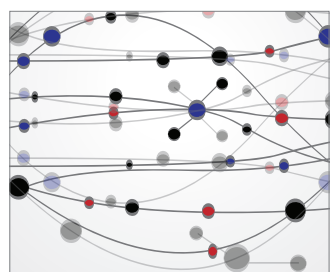

\section{The Scientific} World Journal
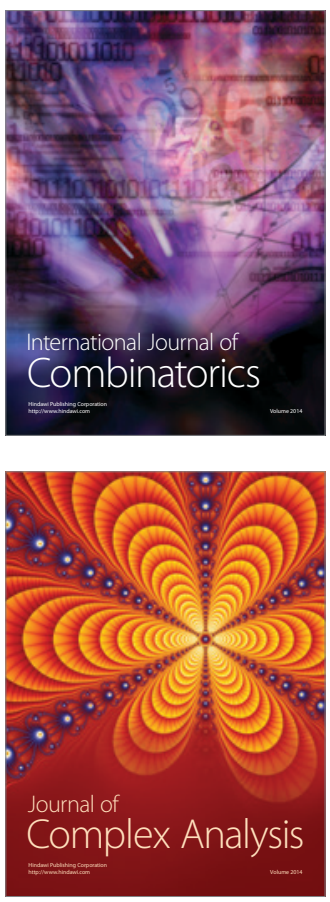

International Journal of

Mathematics and

Mathematical

Sciences
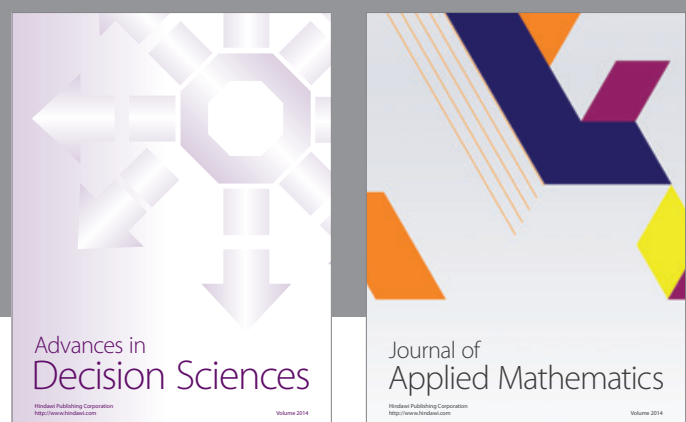

Journal of

Applied Mathematics
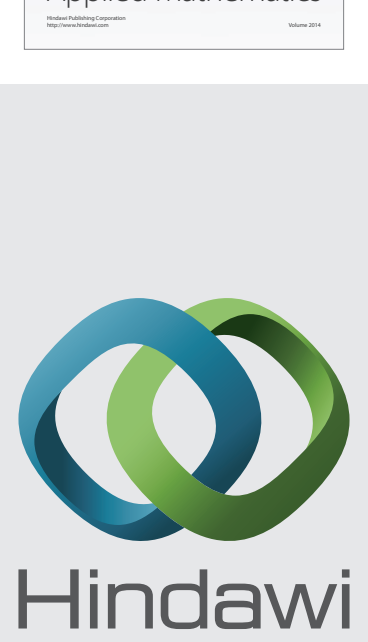

Submit your manuscripts at http://www.hindawi.com
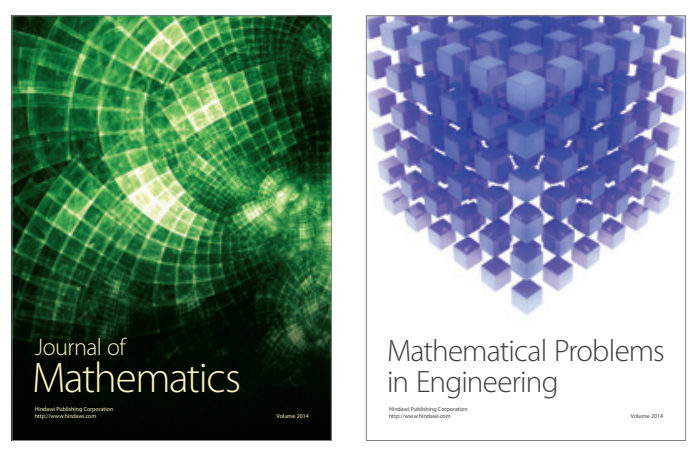

Mathematical Problems in Engineering
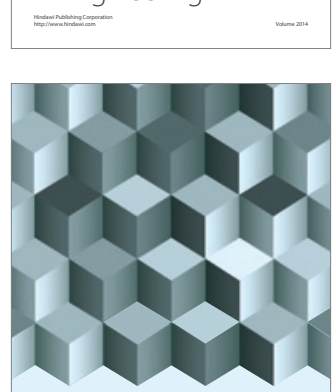

Journal of

Function Spaces
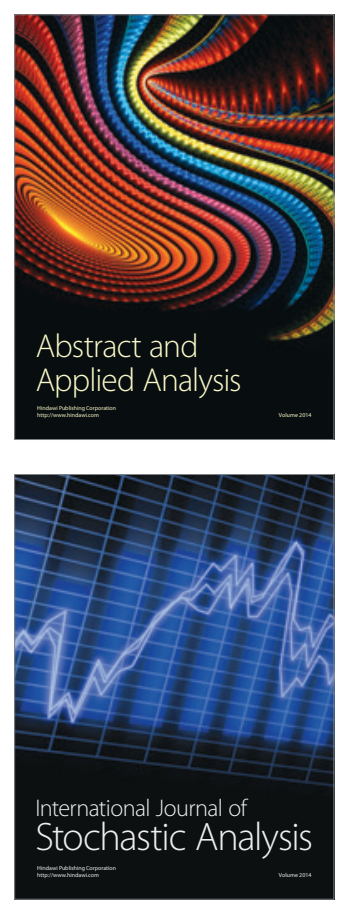

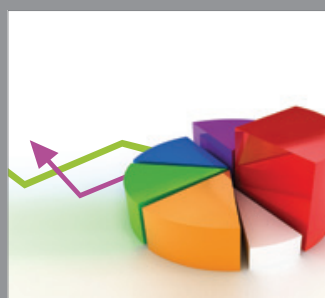

ournal of

Probability and Statistics

Promensencen
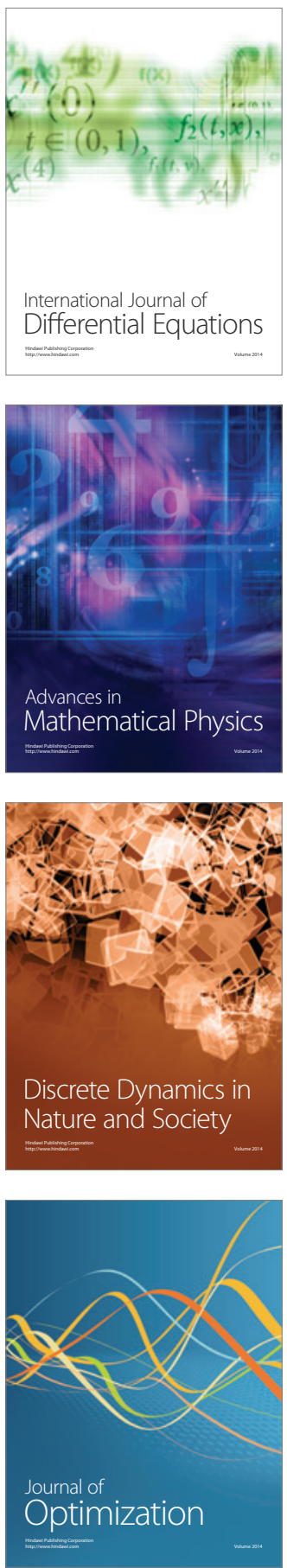